\title{
PERSISTENCIA INFLACIONARIA EN COSTA RICA: PRECIOS DE SERVICIOS Y REGULADOS
}

\author{
Jorge León Murillo ${ }^{1}$ \\ Marco Valerio Berrocal ${ }^{2}$
}

Recibido: 01/08/2014

Aprobado: 13/10/2014

\begin{abstract}
RESUMEN
El documento estudia el grado de persistencia inflacionaria y las rigideces de precios, a nivel desagregado y agregado, de los artículos del Índice de Precios al Consumidor (IPC) en los subíndices de Bienes-Servicios y No regulados-Regulados en Costa Rica. La medición se basa en dos enfoques: el paramétrico se basa en un esquema autorregresivo con media constante y el estructural se basa en la estimación de una Curva de Phillips Neo Keynesiana híbrida. El análisis incluye tanto series de tiempo, como de datos de panel.

La evidencia apunta a que los precios de artículos regulados y servicios poseen mayores grados de persistencia, además de presentar rigideces en su patrón de ajuste. Se encontró que utilizando datos desagregados la persistencia inflacionaria es menor a las estimaciones univariadas con datos agregados, además el nivel de persistencia tiende a reducirse aún más cuando se excluyen los artículos regulados o los servicios del IPC.
\end{abstract}

PALABRAS CLAVE: PERSISTENCIA INFLACIONARIA, PRECIOS RÍGIDOS, PRECIOS REGULADOS, SERVICIOS.

\section{ABSTRACT}

The present document studies the degree of inflation persistence and price stickiness across different categories in the Costa Rican Consumer Price index (CPI), using disaggregate and aggregate data, with a particular focus in the regulated and services prices. We adopt two approaches: The parametric approach is based in an autoregressive process with constant mean, and the structural approach is based on the estimation of the New Keynesian Hybrid Phillips Curve. We use different econometric techniques, as univariate and multivariate time series, and panel data methods.

The evidence suggests that changes in prices and services regulated items have higher degrees of persistence in addition to presenting rigidities in the adjustment pattern. Also it was found that using disaggregate data the degree of inflation persistence is lower than the inflation persistence estimation of univariate models with aggregate data, and this tend to reduce excluding the regulated and services products prices.

KEY WORDS: INFLATION PERSISTENCE, STICKY PRICES, REGULATED PRICES, SERVICES.

1 Banco Central de Costa Rica, Código Postal 10058-1000; Costa Rica; leonmj@bccr.fi.cr

2 Universidad Nacional de Costa Rica, estudiante, Código Postal 11501-2060; Banco Central de Costa Rica, Código Postal 10058-1000; condición de pasante; Costa Rica markxsw@gmail.com 


\section{INTRODUCCIÓN}

El presente documento analiza el grado de persistencia ${ }^{3}$ de la inflación, con un énfasis especial en los artículos del Índice de Precios al Consumidor (IPC) que cuentan con la característica de ser regulados y (o) servicios. La realización de un estudio de la persistencia inflacionaria y las rigideces de precios, en estos subgrupos, pretende mejorar la manera en que se entiende la dinámica inflacionaria del país.

La persistencia inflacionaria y las rigideces de los precios han sido ampliamente estudiadas y existe gran cantidad de literatura enfocada en este tema ${ }^{4}$, esto debido a que la comprensión de la dinámica inflacionaria es de suma importancia para la política económica; porque permite obtener información acerca de la magnitud, duración, y velocidad de ajuste de los precios, lo que permite una mejor planificación en el uso de los instrumentos de política económica para alcanzar un nivel deseado de inflación. "(...) la naturaleza de la dinámica de la inflación, así como la eficacia de la política monetaria, depende en gran medida de las características y los patrones de fijación de precios así como de las rigideces nominales asociadas." (Galí, y otros, 2006).

En los estudios de Altisssimo, Ehrmann y Smets(2006), Bils y Klenow (2002), Lunnemann y Mätha (2005) y Nakamura y Steinsson (2013), se reconoce la interrelación que existe entre la evolución histórica de la inflación, las rigideces de precios y la persistencia de inflación. Los autores encuentran que las rigideces de precios tienden prolongar la persistencia de las variaciones de los precios ante un choque. Para el Caso de Costa Rica no se encontraron estudios que caractericen las rigideces de precios de la economía, por esta razón se decidió calcular indicadores de rigidez de precios para los artículos regulados y servicios del IPC, y así estudiar la relación entre persistencia inflacionaria $y$ rigideces de precios.

Para estimar un parámetro que funcione como una medida del grado de persistencia inflacionaria se realizaron distintas estimaciones. Estas se realizan siguiendo dos enfoques, el paramétrico y el estructural, $y$ distintas metodologías tanto de series de tiempo y datos de panel lo que permite contrastar las diferentes mediciones de persistencia obtenidas, tanto con datos agregados y datos desagregados.

El documento se estructura de la siguiente manera: en la sección 2 se presentan consideraciones teóricas acerca de la persistencia inflacionaria y la curva de Phillips con rigideces "a la Calvo", la cual es la base del enfoque estructural. En la sección 3 se presenta la metodología empleada así como aspectos acerca del manejo de datos. La sección 4 contiene un análisis descriptivo de datos y los resultados de las estimaciones realizadas y por último, en la sección 5, se presentan las principales conclusiones.

\section{CONSIDERACIONES TEÓRICAS}

La persistencia se define como la velocidad con la que una serie de tiempo regresa a su equilibrio (o tendencia de largo plazo) después de ser impactada por una perturbación (Marques, 2004). La medición de la persistencia se puede abordar desde dos enfoques distintos, estos son el estructural y el paramétrico.

El enfoque estructural se basa en la estimación de una Curva de Phillips Neo Keynesiana Híbrida, lo que permite distinguir diferentes tipos de persistencia según cual sea el comportamiento de los agentes que la genere. La forma funcional de la Curva de Phillips Neo Keynesiana Híbrida (CPNKH) viene dada por la ecuación 1. Esta ecuación es derivada a través de micro fundamentos,

3 Se define persistencia como la duración media que tiene un choque sobre una variable.

4 Por ejemplo véase: Altisssimo, Ehrmann y Smets(2006), Babecki(2008), Bihan(2011), Bilke(2005), Galí (2006), Marques(2004), Raciborski(2008) y para el caso específico de Costa Rica Chaverri y Torres(2010). 
la derivación más común es la realizada a través del modelo de Calvo $(1983)^{5}$ con esta derivación se determina que los coeficientes de la CPNKH son una función de los parámetros de impaciencia $(\beta)$, la frecuencia en que cambian los precios (1- $\theta)$ y la elasticidad de la oferta $(\varphi)$. Y además el termino rezagado ( $\delta)$ determina el grado de "inercia" que mantiene el proceso inflacionario.

$$
\begin{gathered}
\pi_{t}=\omega\left(y_{t}-\bar{y}_{t}\right)+\gamma \mathrm{E}\left(\pi_{t+1}\right)+\delta \pi_{t-1}+\varepsilon_{t} \\
\omega=\frac{\varphi(1-\theta)(1-\theta \beta)}{\theta} \\
\varepsilon_{t}=\frac{u_{t}}{\theta} \\
\gamma=(1-\delta) \beta
\end{gathered}
$$

El enfoque estructural predice que el grado de rigidez de precios tiene efectos sobre los niveles de persistencia de inflación, estudios como el de Bils y Klenow (2002) se encuentra que la respuesta de la inflación, ante una perturbación, difiere entre artículos que presentan rigideces de precios y los que no las presentan. Los autores encuentran que las rigideces de precios tienden a reducir la magnitud de las perturbaciones y al mismo tiempo elevan su persistencia.

Con respecto a los tipos de persistencia que permite identificar el enfoque estructural se encuentra la persistencia intrínseca, la persistencia extrínseca y la basada en expectativas. Los grados de persistencia inflacionaria son cuantificados por los coeficientes, $y$ respectivamente.

Por otra parte el enfoque paramétrico se basa en la estimación de un proceso autorregresivo de orden $p$, que resume el comportamiento de la serie de inflación ante distintos choques en el componente residual $\varepsilon_{\mathrm{t}}$.

$$
\pi_{\mathrm{t}}=\alpha+\sum_{\mathrm{j}=1}^{\mathrm{p}} \beta_{\mathrm{j}} \pi_{\mathrm{t}-\mathrm{j}}+\varepsilon_{\mathrm{t}} \text { con } \varepsilon \sim \mathrm{n}(0,1)
$$

"La persistencia inflacionaria $(p)$ se refleja en la suma de los coeficientes autorregresivos significativos de orden p en la ecuación (5)" (Chaverri \& Torres, 2010) de forma que la medida de persistencia de la serie de inflación viene dada por la ecuación 6 ,

$$
\sum_{j=1}^{p} \beta_{j}=\rho
$$

donde $0<p<1$, lo indica que la serie de inflación es estacionaria y cumple con la propiedad de reversión a la media. El cumplimiento de la propiedad de reversión a la media quiere decir que si una perturbación eleva (reduce) el nivel de inflación por encima (debajo) de su valor medio el nivel de inflación disminuirá (aumentará) hasta converger nuevamente a su nivel de equilibrio.

La propiedad de reversión a la media es descrita por la velocidad de convergencia de la serie de tiempo. La velocidad de convergencia a la media de la serie de tiempo $\lambda$ depende inversamente del parámetro $p$, pues $\lambda=1-p$. Cuando el parámetro es muy cercano a 1 se dice que ante una 
perturbación en la variación del índice de precios del bien $i$ es muy persistente dado que su velocidad de convergencia es cercana a 0 , en caso contario cuando el valor es muy cercano 0 la velocidad de convergencia es cercana a 1 y por lo tanto es muy poco persistente. Si el valor es estrictamente 1 significa que una perturbación temporal tendría un efecto permanente sobre la inflación debido a que la variable diverge de su valor de equilibrio.

Siguiendo a Marques (2004) la ecuación 5 se puede expresar como un mecanismo de corrección de desvíos de la inflación con respecto a su valor medio:

$$
\left(\pi_{t}-\mu\right)=\rho\left(\pi_{t-1}-\mu\right)+\sum_{i=1}^{p} \delta_{i} \Delta\left(\pi_{t-i}-\mu\right)
$$

Donde $\mu=\frac{\alpha}{(1-\rho)}$ es la media incondicional de la variable inflación, $y$ se interpreta como el nivel de equilibrio de la inflación. Los parámetros $\delta_{i}$ son los correctores de los desvíos de la inflación con respecto a su valor medio, el signo esperado de estos coeficientes es negativo.

El enfoque univariado para la medición de persistencia presenta algunas dificultades " $E l$ concepto de persistencia está intimamente relacionado con la función de impulso respuesta de un proceso autorregresivo de orden $p$. De cualquier forma, la función de impulso respuesta no es una medida útil de persistencia(...)" (Marques, 2004) además el valor de es una medida abstracta de persistencia lo que dificulta su interpretación, para resolver estas dificultades se utilizan dos medidas que resumen la información contenida en la función de impulso respuesta de la inflación, estas son, la respuesta acumulada a un shock $(R \alpha)$ y la vida media de un choque inflacionario $(\mathrm{Vm})$. La repuesta acumulada viene dada por la ecuación 8 y la vida media de choque por la ecuación 9 .

$$
\begin{gathered}
R a=\sum_{j=0}^{\infty} \frac{\partial \pi_{t+j}}{\partial \varepsilon}=\frac{1}{1-\rho} \\
V m=\frac{\log (0,5)}{\log (\rho)}
\end{gathered}
$$

La vida media se define como el número de periodos en los cuales el efecto de un choque unitario se mantiene por encima de $1 / 2, y$ la respuesta acumulada como el efecto de largo plazo, en puntos porcentuales, que provoca un choque unitario sobre la serie de inflación.

\section{ASPECTOS METODOLÓGICOS Y DE TRATAMIENTO DE DATOS}

\section{Metodología}

Esta sección presenta los distintos indicadores utilizados para determinar el grado de rigidez de precios y luego se presenta el método usado para estimar el nivel de persistencia de las variaciones de los subíndices del IPC.

\section{- Medición de la rigidez de precios}

Con el fin de determinar cuál es la duración promedio en que los precios de un bien $i$ cambian se calcula la frecuencia con la que los precios cambian, la frecuencia se define como el número 
de cambios en los precios por unidad de tiempo ${ }^{6}$; esta medida es el grado de rigidez de los precios. Para cuantificar su magnitud primero se calculan las variaciones mes a mes del índice de precios de cada artículo, luego se genera una variable de conteo denominada $I_{i t} y$ por último para obtener la frecuencia, el valor de 1- $\theta$, se calcula el promedio de la variable de conteo para cada categoría, como muestra la ecuación 10 .

$$
1-\theta=\frac{\sum_{t=1}^{T} I_{i t}}{T}, I_{i t}=\left\{\begin{array}{l}
1 \text { si } p_{i t} \neq p_{i, t-1} \\
0 \text { si } p_{i t}=p_{i, t-1}
\end{array}\right.
$$

Para medir el tipo de rigidez de precios ya sea al aumentar o disminuir (a la baja o al alza) se utilizará el procedimiento propuesto por Kasuya (1999) el cual consiste en calcular el coeficiente de asimetría de las variaciones de los precios para cada artículo de la canasta del IPC. Por otra parte, la distribución de los coeficientes de asimetría, en un corte transversal, de los artículos que cuentan con un grado de rigidez de precios permite aproximar la proporción de artículos del IPC que cuentan con rigidez al alza o bien la proporción de los artículos con un grado de rigidez a la baja en sus precios. Si un determinado artículo del IPC presenta un coeficiente de asimetría positivo implica que los precios de este artículo tienden a aumentar en una mayor magnitud que en la que disminuyen, por lo que tendría un grado de rigidez a la baja en su precios. En caso que el coeficiente sea negativo existen rigideces al alza.

Para calcular la duración en meses en que ocurren cambios en los precios se utilizan dos medidas, la duración promedio y la duración mediana. La duración promedio es igual al inverso de la frecuencia y la duración mediana viene definida por la ecuación 11.

$$
m=-\frac{1}{\ln (\theta)}
$$

Donde $m$ es el número de periodos que transcurren hasta que ocurra una modificación en los precios. La probabilidad de que suceda este evento es la frecuencia promedio en que ocurren los cambios de precios. La ecuación 11 es derivada de una función de duración representada por la ecuación 12, que corresponde a una función de probabilidad exponencial.

$$
1-\theta=s(t)=1-e^{-m}
$$

Si el valor de 1- $\theta$ es igual a 1 esto indica que el precio de ese bien cambió todos los meses por lo que no presenta ninguna rigidez en los cambios de su precio y el valor de no aplica, pero a medida que el valor de $1-\theta$ se acerque a 0 la duración mediana tenderá a $+\infty$ y el precio de este artículo tardará más meses en cambiar su precio, por lo que tiene un grado de rigidez en las variaciones de su precio.

\section{- Modelos univariados de persistencia inflacionaria con datos agregados}

Debido a que existen quiebres estructurales en las variaciones de los índices de precios se calculan distintos valores de $\mu$ "Dado que la media de la inflación varía en el tiempo, si se supone que ésta es constante puede erróneamente concluirse que la inflación es altamente persistente cuando no lo es y viceversa. (...) este problema se enfrenta identificando los quiebres estructurales en el nivel de la serie." (Chaverri \& Torres, 2010).

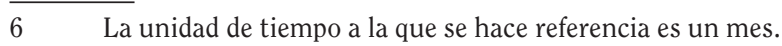


Las fechas de los quiebres estructurales contemplados en esta investigación son julio 2006 y mayo 2009, estos periodos fueron identificados por Torres (2012) el cual utilizó en su estudio pruebas Bai-Perron para múltiples cambios estructurales para índices de precios en la economía de Costa Rica.

Luego de estimar los respectivos valores de, se genera la variable $\left(\pi_{t}-\mu\right)$ y se estima la ecuación 7 con 12 correctores $\delta_{i}$, $y$ secuencialmente se eliminan los que no resulten ser significativos.

Luego de realizar las estimaciones de se determina el grado de persistencia de cada variable, caracterizando las funciones impulso respuesta con las medidas de vida media y respuesta acumulada de un choque inflacionario.

\section{- $\quad$ Modelo multivariado con datos agregados}

Con el fin de obtener un modelo que mida la persistencia inflacionaria en un esquema multivariado se realiza la estimación de un modelo de Vectores Autorregresivos (VAR). Esto permite determinar la magnitud $y$ persistencia de los choques inflacionarios que se transmiten entre los componentes del IPC.

En general un vector autorregresivo viene dado por la ecuación:

$$
z_{t}=\beta_{0}+\sum_{\mathrm{k}=1}^{\mathrm{K}} \beta_{\mathrm{k}} \mathrm{z}_{\mathrm{t}-\mathrm{k}}+\sum_{\mathrm{j}=1}^{\mathrm{J}} \delta_{\mathrm{j}} \mathrm{x}_{\mathrm{t}-\mathrm{j}}+\varepsilon_{\mathrm{t}}
$$

Donde $X$ es un vector de variables exógenas, $Z$ es un vector son las variables endógenas y $\varepsilon_{\mathrm{t}} \sim \mathrm{N}\left(0, \sum\right)$ es un vector de shocks aleatorios.

El atractivo de los modelos VAR es que una vez que se estimado es posible realizar las simulaciones de las variables endógenas midiendo la respuesta del sistema ante perturbaciones, la distribución de estas perturbaciones la realiza la factorización de Cholesky:

$$
\varepsilon_{t}=W v_{t}
$$

Dónde:

W: Es resultado de la factorización de Cholesky de la matriz $\sum$, tal que $\sum=\mathrm{WW}^{\prime}$

$v_{t}$ : Es un vector de shocks tal que $v_{t} \sim N(0,1)$

La matriz $\mathrm{W}$ es obtenida mediante la factorización de Cholesky de la matriz de varianzas-covarianzas, esta permite que los shocks se propaguen por el sistema de ecuaciones del VAR. Al definir un impulso utilizando la factorización de Cholesky es crucial definir el orden de las variables ya que esto determina la dinámica del sistema, por lo que las variables son ordenadas según su grado de exogeneidad.

La medición de persistencia en un esquema multivariado se realizará caracterizando las funciones de impulso respuesta para esto se analizará la duración de los efectos significativos, ya que describe la duración de los choques en el sistema. Además se utilizarán las medidas de respuesta acumulada $y$ vida media de un choque inflacionario para caracterizar las funciones de impulso respuesta.

\section{- $\quad$ Modelos con datos desagregados}

Para medir el nivel de persistencia inflacionaria a nivel desagregado se utiliza el IPC por artículo y se estiman modelos con técnicas de datos de panel. Se estiman dos especificaciones, una 
basada en la Curva de Phillips Neo Keynesiana Híbrida y otra basada en el esquema paramétrico de reversión a la media propuesto por Marques (2004). El modelo basado en la Curva de Phillips sigue la siguiente especificación:

$$
\begin{gathered}
\pi_{i, t}=\beta_{1 i}+\beta_{2 i} \pi_{i, t-1}+\beta_{3 i} E\left(\pi_{t}\right)+\beta_{4 i}\left(y_{t}-\bar{y}_{t}\right)+\varepsilon_{i t} \\
\varepsilon_{i t}=a_{i}+v_{i t}
\end{gathered}
$$

dónde $\pi_{t}^{i}$ es la variación del precio de cada artículo " $i$ ” en un periodo, $E\left(\pi_{t}\right)$ es la expectativa de inflación, $y y_{t}-\bar{y}_{t}$ es la brecha del producto. La ventaja de esta especificación funcional es que permite identificar el grado de distintos tipos de persistencia, el coeficiente asociado al proceso autorregresivo $\beta_{2 i}$ permite medir el grado de persistencia intrínseca, el parámetro $\beta_{4 i}$ mide el grado de persistencia extrínseca y el parámetro $\beta_{3 i}$ mide el grado de persistencia basada en expectativas. Para todos los tipos de persistencia una mayor magnitud en el parámetro encargado de su medición implica un mayor grado de persistencia.

El modelo basado en el esquema de Marques (2004) es una versión de la ecuación 7, desagregando por artículo “i”, y su interpretación es la misma que la propuesta en el apartado teórico.

$$
\begin{gathered}
\left(\pi_{i t}-\mu_{i}\right)=\rho_{i}\left(\pi_{i, t-1}-\mu_{i}\right)+\sum_{j=1}^{p} \delta_{i} \Delta\left(\pi_{i, t-j}-\mu_{i}\right)+u_{i t} \\
u_{i t}=a_{i}+v_{i t}
\end{gathered}
$$

Al utilizar datos desagregados se puede estimar la persistencia $\left(p_{i}\right)$ para cada artículo del IPC, esto permite identificar los artículos "causantes" de la persistencia de la inflación y además elimina el sesgo de agregación ${ }^{7}$ en las estimaciones.

Se aplicaron distintos métodos de estimación a las ecuaciones 15 y 17: Métodos lineales como efectos fijos y aleatorios; Métodos dinámicos como el estimador de Generalizado de momentos (GMM) de Arellano y Bond en uno y dos pasos; la estimación de regresiones individuales. Para agregar los resultados de las estimaciones individuales se calcula el promedio ponderado utilizando los ponderadores del IPC.

$$
\rho=\sum_{i=1}^{n} \omega_{i} \rho_{i}
$$

Donde son los ponderadores de cada artículo del IPC.

\section{Datos}

Las fuentes de los datos utilizados en esta investigación son el Instituto Nacional de Estadística y Censos (INEC), y el Banco Central de Costa Rica. Se utiliza la información del Índice de Precios al Consumidor en su versión desagregada por artículo, además de los índices de precios Regulados - No regulados y Bienes - Servicios. La muestra utilizada en el documento comprende de enero 1995

$7 \quad$ Estudios como el de Lunnemann (2004) encuentran que los datos agregados presentan mayores grados de persistencia. 
hasta junio del 2013. Debido a que existen diferentes cambios estructurales, identificados por Torres (2012) y cambios metodológicos realizados por el INEC en la medición del IPC en el 2006, en algunas estimaciones se restringe la muestra a periodos donde no existan cambios estructurales o bien a periodos donde estas no afecten la estabilidad de las estimaciones. En el caso que el enlace del IPC 1995 al IPC 2006 distorsione el cálculo de indicadores se utilizará la muestra a partir de julio del 2006.

Las series no fueron tratadas con filtros de estacionalidad siguiendo a Rodríguez (2012) debido a que las estimaciones con series desestacionalizadas sobrestiman el efecto de la persistencia de la inflación y distorsionan los resultados. En estudios como el de Ghysels(1990) y luego Ghysels y Perron(1993) se ha determinado que los filtros de estacionalidad, como el X-11 ARIMA, agregan un sesgo positivo a las estimaciones de coeficientes asociados a un término autorregresivo, y reducen la potencia de las pruebas de raíz unitaria.

Además siguiendo la posición de Sargent (1993) desestacionalizar provoca una pérdida de información considerable; los agentes forman sus expectativas utilizando datos sin desestacionalizar, por lo que las series destacionalizadas pueden sesgar las estimaciones o hacer que estas pierdan eficiencia.

\section{RESULTADOS}

En el siguiente apartado se analizará el comportamiento de las variaciones interanuales de los subíndices que componen el Índice General de Precios subdividiendo la muestra en distintos periodos identificados como quiebres estructurales por Torres (2012), luego de esto se continuará con el cálculo del nivel de rigidez de precios a nivel de artículo, y se buscarán patrones estacionales en los ajustes de los precios. Y por último se muestran las estimaciones de los modelos univariados, multivariados y de datos de panel, utilizados para medir el grado de persistencia inflacionaria.

\section{Comportamiento de los índices de inflación}

Con el fin de analizar el comportamiento de las series en los diferentes quiebres estructurales identificados por Torres (2012) se dividió la muestra en 3 periodos los cuales comprenden de diciembre 1996 a junio 2006, de julio 2006 a abril 2009, y de mayo 2009 a junio 2013. Para los periodos en que se dividió la muestra se estimaron los promedios y desviaciones estándar de los diferentes subíndices que componen el IPC.

El cuadro 1 resume los niveles promedio y desviaciones estándar de las variaciones interanuales de los subíndices de precios, los resultados muestran que los niveles y variabilidades de las variaciones interanuales del IPC se han reducido a partir del último quiere estructural identificado por Torres (2012). 
CUADRO 1

ESTADÍSTICOS DESCRIPTIVOS DE LAS SERIES

\begin{tabular}{|c|c|c|c|c|c|c|c|}
\hline Índice: & General & Regulados & $\begin{array}{l}\text { No } \\
\text { regulados }\end{array}$ & Servicios & Bienes & $\begin{array}{l}\text { Servicios } \\
\text { regulados }\end{array}$ & $\begin{array}{c}\text { Servicios } \\
\text { no } \\
\text { regulados }\end{array}$ \\
\hline \multicolumn{8}{|c|}{ Periodo completo } \\
\hline Media & 10,27 & 11,43 & 9,54 & 11,17 & 9,58 & 11,56 & 11,05 \\
\hline Desvío estándar & 3,61 & 5,45 & 3,35 & 3,21 & 4,49 & 5,65 & 3,48 \\
\hline \multicolumn{8}{|c|}{ Periodo $1996 \mathrm{~m} 12-2006 \mathrm{~m} 6$} \\
\hline Media & 11,40 & 13,25 & 11,09 & 12,11 & 11,02 & 12,46 & 11,97 \\
\hline Desvío estándar & 1,79 & 5,13 & 1,93 & 2,01 & 2,44 & 5,59 & 2,44 \\
\hline \multicolumn{8}{|c|}{ Periodo $2006 \mathrm{~m} 7-2009 \mathrm{~m} 4$} \\
\hline Media & 11,38 & 11,38 & 11,36 & 11,75 & 11,22 & 11,52 & 11,74 \\
\hline Desvío estándar & 2,26 & 4,23 & 2,05 & 2,15 & 3,00 & 4,33 & 1,52 \\
\hline \multicolumn{8}{|c|}{ Periodo $2009 \mathrm{~m} 5-2013 \mathrm{~m} 6$} \\
\hline Media & 5,23 & 7,29 & 4,71 & 7,25 & 3,31 & 8,61 & 6,78 \\
\hline Desvío estándar & 1,09 & 4,56 & 1,41 & 1,99 & 1,47 & 5,72 & 1,52 \\
\hline
\end{tabular}

Fuente: Elaboración propia.

Los grupos de artículos que presentan variaciones interanuales promedio mayores son los regulados, servicios y servicios regulados, además estos grupos se caracterizan por tener medidas de variabilidad mayores.

En el Gráfico 1 se puede observar que la inflación general sigue un comportamiento similar a la inflación de los artículos no regulados, esto debido a que en su construcción los no regulados tienen una ponderación mayor, pero por otra parte las variaciones de los precios de los artículos regulados tienen un comportamiento más volátil.

\section{GRÁFICO 1}

VARIACIÓN INTERANUAL DE ÍNDICES DE PRECIOS: 1996M1-2013M6

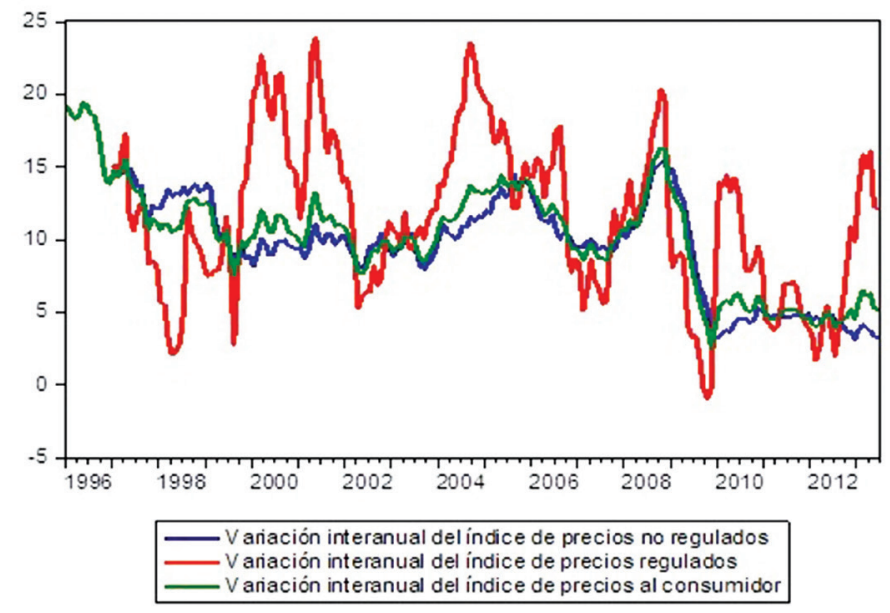

Fuente: Elaboración propia. 
La mayor volatilidad del componente regulado se puede deber a arreglos institucionales que existen en la agencia reguladora del bien o servicio correspondiente. Lo cual acumula ajustes de precios, es decir ejecuta incrementos de precios de mayor magnitud a periodicidades más bajas, o bien a que la metodología de ajustes de precios sobre reacciona ante las variaciones de los precios no regulados.

Observando los subíndices de bienes y servicios (Gráfico 2) se identificó que existe un comportamiento divergente entre las dos series a partir de enero del 2013, donde los servicios comienzan a presentar tasas de variación interanuales mayores que los bienes. Esta divergencia se puede atribuir en parte a las variaciones del componente de servicios regulados y otros ajustes en los precios servicios que se dan estacionalmente, que son exógenos para el Banco Central:

"La variación mensual del IPC en febrero, al igual que el mes previo, estuvo asociada en mayor medida a factores exógenos al Banco Central. Entre estos destacaron los choques de oferta de origen agrícola y de hidrocarburos, así como la marcada estacionalidad al alza de los servicios de educación.

En términos de la tasa interanual, los bienes y servicios regulados, con una ponderación de $20 \%$ en la canasta del IPC, aportaron el 50\%. En lo referente al componente no regulado, destacaron las alzas en el servicio de educación formal y los bienes agrícolas, con una contribución del 7\% y 6\%, respectivamente." (Informe mensual de coyuntura macroeconómica del BCCR, marzo 2013)

GRÁFICO 2

INFLACIÓN INTERANUAL: BIENES Y SERVICIOS 1996M1-2013M6

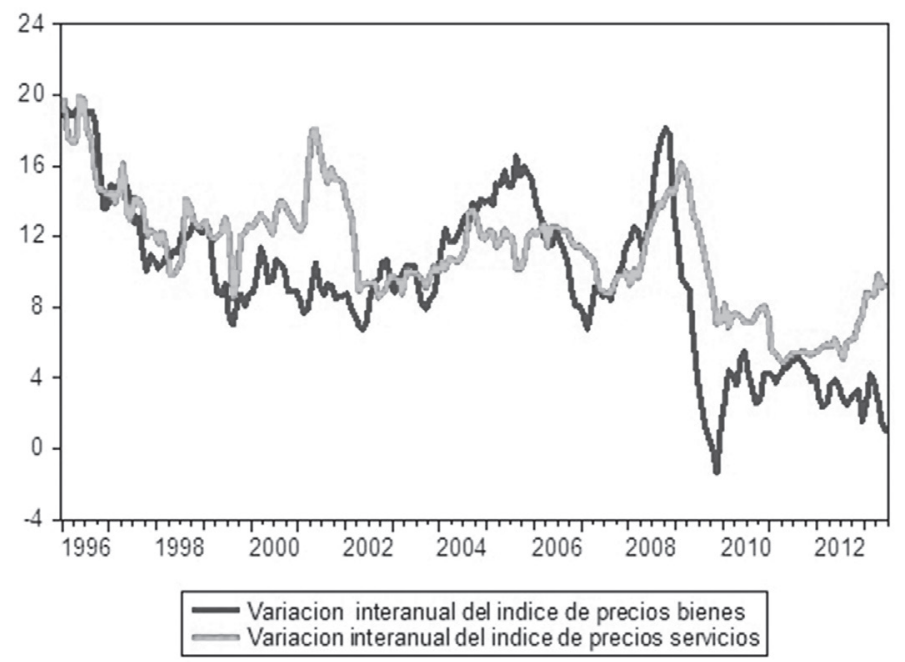

Fuente: Elaboración propia.

2. Rigidez de precios por grupo de artículos

Siguiendo la metodología de Bils y Klenow (2002) se calculó el nivel de rigidez de precios de cada artículo del IPC, esto basado en la duración promedio en que tardan en cambiar los precios. Luego de esto se estudió las diferencias entre las medias de los indicadores según grupos de artículos. 
Al aplicar una prueba de diferencias de medias se observó que los precios de los artículos regulados son significativamente más rígidos que los precios no regulados, estos exhiben una duración promedio de 1,85 meses y 1,09 meses, respectivamente. También se encontró que los precios de los servicios son, significativamente, en promedio más rígidos que los bienes con una duración promedio de 1,57 meses y 1,03 meses respectivamente. Los artículos que tienen la característica de ser servicios regulados presentan una mayor rigidez promedio que el resto de los artículos, con una duración de 2,66 meses.

La medida de magnitud de cambio de los precios, promedio del valor absoluto de las variaciones mes a mes e interanual, no presenta diferencias de medias significativas entre los grupos de bienes y servicios, regulados $y$ no regulados.

La correlación entre la frecuencia y magnitud de los cambios de los precios por artículo es positiva $y$ muy baja $0,14^{8}$.

Mediante el cálculo de la distribución de los coeficientes de asimetría de las variaciones de los precios de cada artículo se estima que aproximadamente el 74\% de los artículos que presentan algún grado de rigidez de precios presentan rigidez a bajar sus precios.

\section{GRÁFICO 3 \\ DISTRIBUCIÓN DE LOS COEFICIENTES DE ASIMETRÍA DE LAS VARIACIONES DE LOS PRECIOS DE CADA ARTÍCULO QUE PRESENTA ALGUN NIVEL DE RIGIDEZ DE PRECIOS}

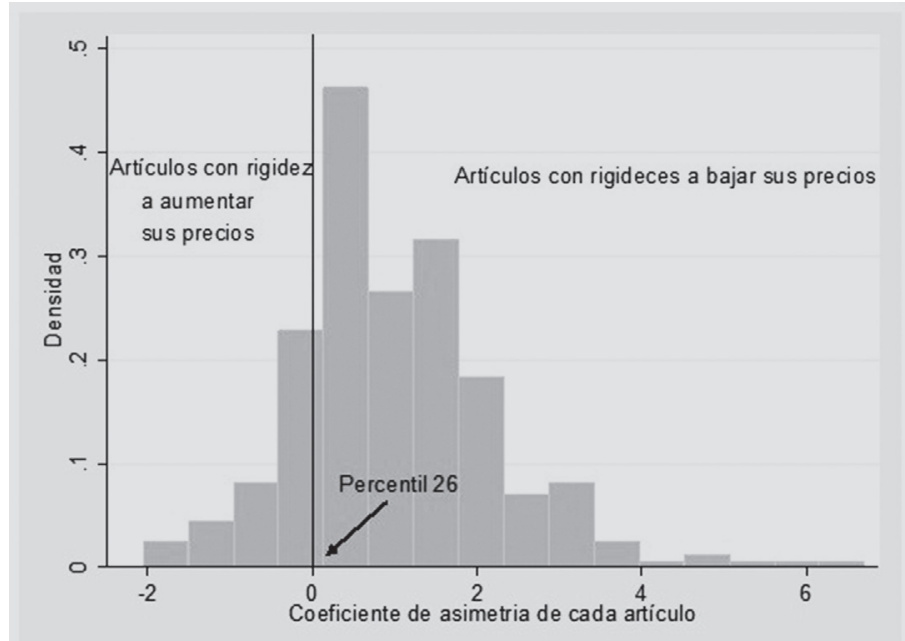

Fuente: Elaboración propia.

El Cuadro 2 muestra los 20 artículos con precios más rígidos del IPC. Cabe resaltar que los artículos más rígidos son servicios regulados. Los artículos con precios más flexibles, en general, son bienes que no están sujetos a ninguna regulación y cuyos precios cambian en todas las observaciones.

8 Un autor que encuentra correlación muy baja es López (2008). Estos resultados son contrarios a los obtenidos por Lunnemann(2005). 
CUADRO 2

LOS 20 ARTÍCULOS CON PRECIOS MÁS RÍGIDOS

\begin{tabular}{|c|c|c|c|c|c|c|}
\hline \# & ARTÍCULO & Frecuencia & $\begin{array}{c}\text { Duración } \\
\text { promedio } \\
\text { (meses) }\end{array}$ & $\begin{array}{c}\text { Duración } \\
\text { mediana } \\
\text { (meses) }\end{array}$ & Servicio & Regulado \\
\hline 1 & Licencia de conducir & 0,01 & 84,00 & 83,50 & $\mathrm{x}$ & $\mathrm{x}$ \\
\hline 2 & Revisión técnica de vehículo & 0,01 & 84,00 & 83,50 & $\mathrm{x}$ & $\mathrm{X}$ \\
\hline 3 & Servicio de teléfono fijo & 0,01 & 84,00 & 83,50 & $\mathrm{x}$ & $\mathrm{X}$ \\
\hline 4 & Servicio de teléfono celular & 0,01 & 84,00 & 83,50 & $\mathrm{x}$ & $\mathrm{X}$ \\
\hline 5 & Periódicos & 0,07 & 14,00 & 13,49 & & \\
\hline 6 & Honorarios a abogado & 0,10 & 10,50 & 9,99 & $\mathrm{x}$ & \\
\hline 7 & Educación primaria & 0,23 & 4,42 & 3,90 & $\mathrm{x}$ & \\
\hline 8 & Educación secundaria & 0,24 & 4,20 & 3,68 & $\mathrm{x}$ & \\
\hline 9 & Educación preescolar & 0,25 & 4,00 & 3,48 & $\mathrm{x}$ & \\
\hline 10 & Entrada al cine & 0,31 & 3,23 & 2,70 & $\mathrm{x}$ & \\
\hline 11 & Anteojos graduados & 0,33 & 3,00 & 2,47 & $\mathrm{x}$ & \\
\hline 12 & Transporte en taxi & 0,35 & 2,90 & 2,36 & $\mathrm{x}$ & $\mathrm{X}$ \\
\hline 13 & Alquiler de película & 0,36 & 2,80 & 2,26 & $\mathrm{x}$ & \\
\hline 14 & Texto universitario & 0,40 & 2,47 & 1,93 & & \\
\hline 15 & Servicios veterinarios & 0,45 & 2,21 & 1,66 & $\mathrm{x}$ & \\
\hline 16 & Parqueo público & 0,45 & 2,21 & 1,66 & $\mathrm{x}$ & \\
\hline 17 & Educación superior & 0,45 & 2,21 & 1,66 & $\mathrm{x}$ & \\
\hline 18 & Servicio de pintura para vivienda & 0,48 & 2,10 & 1,55 & $\mathrm{x}$ & \\
\hline 19 & Salveque & 0,48 & 2,10 & 1,55 & & \\
\hline 20 & Servicio de agua & 0,50 & 2,00 & 1,44 & $\mathrm{x}$ & $\mathrm{X}$ \\
\hline
\end{tabular}

Fuente: Elaboración propia.

\section{Patrones estacionales en los cambios de los precios}

El Cuadro 3 muestra las variaciones mes a mes promedio observadas en el periodo 2009m5-2013m6. Las variaciones mes a mes del IPC son mayores en los meses de enero y diciembre. Desagregando por subíndices se observa que los artículos regulados y en especial los servicios regulados tienden a aumentar de precios en mayor magnitud en los meses enero, abril, agosto y 
noviembre. Los artículos no regulados tienen variaciones mayores en febrero julio y diciembre. Además se identifica que los bienes tienen, en promedio, mayores tasas de inflación en diciembre y los servicios en enero.

CUADRO 3

VARIACIONES MENSUALES PROMEDIO: PATRONES ESTACIONALES (2009M5- 2013M6)

\begin{tabular}{lcccccc}
\hline & Enero & Febrero & Marzo & Abril & Mayo & Junio \\
\hline General & 0,95 & 0,61 & 0,25 & 0,38 & 0,41 & 0,23 \\
Regulados & $\underline{1,96}$ & 0,25 & 0,95 & $\underline{1,43}$ & 1,07 & 0,47 \\
No regulados & 0,68 & $\underline{0,70}$ & 0,07 & 0,10 & 0,24 & 0,17 \\
Servicios & $\underline{1,71}$ & 0,96 & 0,34 & 0,34 & 0,49 & 0,38 \\
Servicios regulados & $\underline{2,88}$ & 0,11 & 0,22 & 0,40 & 1,23 & 0,40 \\
Servicios no regulados & $\underline{1,27}$ & $\underline{1,28}$ & 0,39 & 0,30 & 0,23 & 0,37 \\
Bienes & 0,19 & 0,26 & 0,16 & 0,42 & 0,33 & 0,08 \\
\hline & Julio & Agosto & Septiembre & Octubre & Noviembre & Diciembre \\
\hline General & 0,32 & 0,41 & $-0,01$ & 0,35 & 0,40 & 0,74 \\
Regulados & 0,09 & $\underline{0,76}$ & 0,11 & 0,49 & $\underline{0,62}$ & 0,27 \\
No regulados & $\underline{0,39}$ & 0,32 & $-0,05$ & 0,31 & 0,34 & $\underline{0,87}$ \\
Servicios & 0,23 & 0,54 & 0,16 & 0,38 & 0,48 & 0,68 \\
Servicios regulados & $-0,32$ & 1,07 & 0,00 & 0,77 & 0,98 & 0,75 \\
Servicios no regulados & 0,43 & 0,35 & 0,21 & 0,24 & 0,30 & 0,66 \\
Bienes & 0,41 & 0,28 & $-0,19$ & 0,31 & 0,32 & $\underline{0,78}$ \\
\hline
\end{tabular}

Fuente: Elaboración propia.

\section{Modelos univariados: Persistencia inflacionaria en los subíndices de precios}

El cuadro 5 resume los resultados de las estimaciones realizadas de la medida de persistencia inflacionaria además de la vida media de los choques y la respuesta acumulada de la variable ante un choque unitario. Las categorías que muestran una mayor persistencia inflacionaria son los bienes, y servicios no regulados, las categorías que presentan menor persistencia son los servicios regulados que representan $80 \%$ de la canasta de los artículos regulados. 
CUADRO 4

PERSISTENCIA INFLACIONARIA POR SUBÍNDICE

\begin{tabular}{|c|c|c|c|c|c|c|}
\hline & \multirow{2}{*}{$\begin{array}{c}\text { Coeficiente } \\
p\end{array}$} & $\begin{array}{c}\text { Vida } \\
\text { media }\end{array}$ & $\begin{array}{c}\text { Respuesta } \\
\text { acumulada }\end{array}$ & Autocorrelación & Homocedasticidad & Normalidad \\
\hline & & (Meses) & (pp) & \multicolumn{3}{|c|}{ (P-value) } \\
\hline General & 0,2939 & 0,57 & 1,42 & 0,3406 & 0,3697 & 0,0341 \\
\hline Regulados & 0,2427 & 0,49 & 4,88 & 0,1624 & 0,2795 & $0,0000^{*}$ \\
\hline No regulados & 0,2580 & 0,51 & 5,10 & 0,4562 & 0,6161 & 0,3919 \\
\hline Servicios & 0,1512 & 0,37 & 3,66 & 0,0410 & 0,0836 & $0,0000^{*}$ \\
\hline Servicios regulados & 0,1134 & 0,32 & 3,17 & 0,8654 & 0,4855 & $0,0000^{*}$ \\
\hline Servicios no regulados & 0,1473 & 0,36 & 3,61 & $0,0000^{*}$ & 0,1942 & $0,0000^{*}$ \\
\hline Bienes & 0,3367 & 0,32 & 6,35 & 0,6985 & $0,0088^{*}$ & $0,0003^{*}$ \\
\hline
\end{tabular}

*Significativos al 1\%

Fuente: Elaboración propia con base en las estimaciones realizadas.

En el caso de los ajustes de los precios de los artículos regulados y servicios se encontró una baja persistencia. Debido a que las variaciones de los precios de los artículos regulados ocurren en el periodo que el regulador defina y luego no hay cambios, es lógico pensar que un choque en los precios de los regulados no genere una respuesta muy persistente. En el caso de los servicios sucede algo similar ya que generalmente los precios de los servicios se ajustan a inicio de año.

El ajuste escalonado de los precios de los artículos regulados y servicios es la razón de que no exista evidencia estadística para aceptar la hipótesis de normalidad en sus residuos a un nivel de significancia del $1 \%$.

La medida univariada de persistencia es poco informativa sobre las interrelaciones que determinan la persistencia de las variaciones de los índices de precios, por lo que se procede a estimar un modelo multivariado.

\section{Modelo multivariado: Vector autorregresivo}

Las ecuaciones del VAR son estimadas en el espíritu de aproximar una curva de Phillips Neo Keynesiana Híbrida para las variables de inflación, una ecuación de generación de expectativas y una curva IS. Esto nos permite medir los grados de persistencia inflacionaria ya sea intrínseca, extrínseca o basada en las expectativas de los agentes. Cabe resaltar que un modelo VAR no es una representación de la estructura de la economía y su carácter es ateórico.

La especificación del Vector Autorregresivo se realiza con la intención de cuantificar el impacto que tendría una perturbación en los precios de los artículos regulados en el sistema de ecuaciones. Por lo que el VAR propuesto en la ecuación 13 se estima con los vectores $z_{t}$ y $x_{t}$ a continuación propuestos ${ }^{9}$ :

$$
z_{t}=\left(\begin{array}{c}
\pi_{t}^{R} \\
E\left(\pi_{t}\right) \\
y_{t}-\bar{y}_{t} \\
\pi_{t}^{N R}
\end{array}\right) \quad x_{t}=\left(T P M_{t}\right)
$$

$9 \quad$ Para determinar el orden de las variables se utilizaron pruebas de exogeneidad de Granger, las pruebas se encuentran en el anexo 1. 
Dónde:

$\pi_{t}^{R}$ : Es la variación interanual del subíndice de precios regulados.

$E\left(\pi_{t}\right)$ : Es la expectativa de inflación a 12 meses.

$y_{t}-\bar{y}_{t}$ : Es la brecha del producto calculada como la diferencia logarítmica del valor observado $y$ la tendencia ${ }^{10}$ de la serie del IMAE tendencia-ciclo.

$\pi_{t}^{N R}$ : Es la variación interanual del subíndice de precios no regulados.

$T P M_{t}$ : Es el promedio mensual de la tasa de interés de política monetaria.

Las pruebas estadísticas de heterocedasticidad, autocorrelación y normalidad, realizadas al modelo VAR se aplicaron suponiendo una probabilidad 1\% de cometer un error tipo 1. Las pruebas muestran, en manera muy general, los siguientes resultados ${ }^{11}$ :

El número de rezagos óptimos del VAR se determinó en 2, con la ayuda del criterio de Shwarz. Todas las raíces características del modelo se encuentran dentro del círculo unitario, por lo que el modelo cumple la condición de estabilidad. Utilizando la prueba de Doornik-Hansen se encontró que los residuos se distribuyen normalmente, esto valida el supuesto de normalidad realizado por otras pruebas estadísticas e incrementa la potencia de las mismas. Utilizando la prueba de White se determinó que no hay suficiente evidencia estadística para rechazar la hipótesis nula de homocedasticidad en los residuos. Aplicando la prueba LM se encuentra evidencia de autocorrelación en el séptimo rezago.

\section{- $\quad$ Análisis de las funciones de impulso-respuesta}

Las funciones de impulso respuesta se presentan en el anexo 3, 4 y 5. Los principales resultados se presentan a continuación.

Un choque de una desviación estándar en la variación interanual del subíndice de precios regulados genera efectos significativos en la brecha del producto y en la variación interanual del subíndice de precios no regulados. Los efectos sobre la brecha del producto comienzan a ser significativos en el quinto mes después del choque inicial y se mantienen así hasta el octavo mes, alcanzando un efecto máximo en el séptimo mes de -0,019pp. Los efectos significativos de un choque de los precios regulados sobre las variaciones interanuales del subíndice de precios no regulados se dan en el tercer mes y se mantienen significativos hasta el cuarto mes, con un efecto máximo de $0,22 \mathrm{pp}$, que representa un $11 \%$ del choque inicial. Este choque se mantiene significativo hasta 4 meses después del choque inicial.

Un choque de una desviación estándar en las expectativas de inflación causa una respuesta de $0,26 \mathrm{pp}$ sobre la variación interanual de los precios no regulados, como efecto máximo en el cuarto mes, el efecto de este choque se mantiene significativo durante cinco meses, del segundo mes hasta el séptimo mes después del impacto inicial. Este choque no se propaga a las variables brecha del producto $y$ variación interanual del subíndice de precios regulados.

Un choque de una desviación estándar en la brecha del producto no se propaga a las demás variables del sistema de ecuaciones.

$\overline{10} \quad$ El cálculo de la tendencia es realizado con el filtro de Hodrick-Prescott con un "Lamda" de 23000, el valor es calculado para la economía de Costa Rica por Segura y Vázquez (2011). 
Además se encontró que un choque de una desviación estándar en la variación interanual del subíndice de precios no regulados tiene un efecto significativo, en el cuarto mes, sobre las variaciones interanuales de precios regulados en una magnitud de $-0,8 \mathrm{pp}$. Este choque es significativo hasta el tercer mes después del choque inicial.

Por último los resultados agregados (Ponderando las respuestas de los componentes del IPC) muestran que un choque en las expectativas, brecha del producto no tienen efectos significativos sobre las variaciones IPC. Por otra parte se encontró que un choque en el componente regulado del IPC causa una respuesta de una magnitud máxima de 0,61pp, en el segundo periodo, sobre la variación interanual del IPC. Este choque tiene efectos significativos que se mantienen hasta el cuarto mes después del choque inicial. Un choque en la variación de los precios no regulados tiene un efecto significativo en el primer mes, con un efecto máximo de 0,30pp. Contando los periodos que tardan las respuestas del IPC en converger se encontró que los choques en los precios regulados y en las expectativas tienen respuestas más persistentes que los choques en las variaciones interanuales de los precios no regulados. 


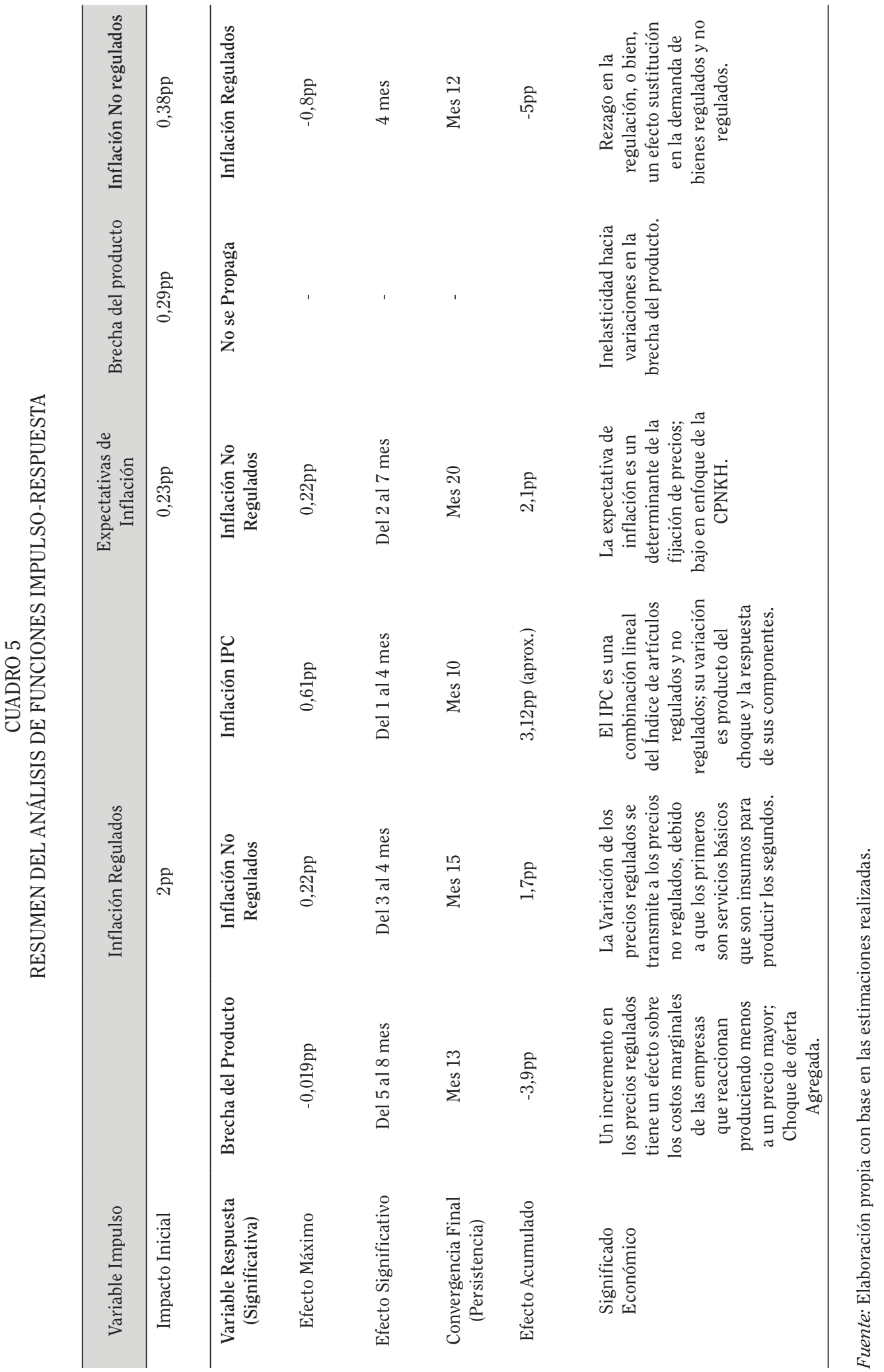




\section{- Descomposición de la varianza}

La descomposición de la varianza permite averiguar el porcentaje de variabilidad que atribuye cada variable explicativa a la variable dependiente, a partir del periodo 10 los valores comienzan a converger por lo que se toma ese periodo como referencia. La descomposición de la varianza en el periodo 10 se presenta en el cuadro $6^{12}$.

\section{CUADRO 6 \\ DESCOMPOSICIÓN DE LA VARIANZA}

\begin{tabular}{|c|c|c|c|c|}
\hline \multirow[b]{2}{*}{ Variable dependiente } & \multicolumn{4}{|c|}{ Variables explicativas } \\
\hline & $\begin{array}{l}\text { Variación interanual } \\
\text { del subíndice de precios } \\
\text { regulados }\end{array}$ & $\begin{array}{l}\text { Brecha del } \\
\text { producto }\end{array}$ & $\begin{array}{l}\text { Expectativa } \\
\text { de inflación }\end{array}$ & $\begin{array}{c}\text { Variación interanual del } \\
\text { subíndice de precios no } \\
\text { regulados }\end{array}$ \\
\hline $\begin{array}{l}\text { Variación interanual del } \\
\text { subíndice de precios regulados }\end{array}$ & $83,96 \%$ & $0,59 \%$ & $3,07 \%$ & $12,38 \%$ \\
\hline Brecha del producto & $37,89 \%$ & $47,98 \%$ & $8,03 \%$ & $0,09 \%$ \\
\hline Expectativa de inflación & $14,74 \%$ & $5,80 \%$ & $78,26 \%$ & $1,28 \%$ \\
\hline $\begin{array}{l}\text { Variación interanual del } \\
\text { subíndice de precios no } \\
\text { regulados }\end{array}$ & $28,32 \%$ & $2,92 \%$ & $38,65 \%$ & $30,11 \%$ \\
\hline
\end{tabular}

Fuente: Elaboración propia con base en las estimaciones realizadas.

La variabilidad de la variación interanual del subíndice de precios regulados, es explicada por un porcentaje considerable por la variación interanual del subíndice de precios de los artículos no regulados. Se encuentra que la variación interanual del subíndice de precios regulados aporta una magnitud considerable de variabilidad a las variables del sistema de ecuaciones.

La descomposición de la varianza además indica que la variación interanual del subíndice de precios no regulados se explica principalmente por la expectativa de inflación, luego por la variación interanual del subíndice de precios regulados. El 28,32\% y 38,65\% de la variabilidad de la variación interanual del subíndice de precios no regulados es explicada por las variables variación interanual del subíndice de precios regulados y expectativa de inflación, respectivamente. El 30,11\% de la variabilidad es explicada por la variación interanual del subíndice de precios no regulados y el 2,92\% restante de la varianza se explica por las variaciones de la brecha del producto.

\section{Modelos de datos de panel}

Las estimaciones realizadas mediante métodos de datos de panel se presentan en esta sección, los resultados se resumen en los cuadros 6,7 y 8 , las estimaciones se realizaron con errores estándar robustos; para aumentar la eficiencia de los estimadores ante posibles problemas en los residuos.

El cuadro 7 muestra los resultados de las estimaciones de la especificación estructural, además del cálculo de la respuesta acumulada de la variable inflación ante un choque unitario y su ajuste medio.

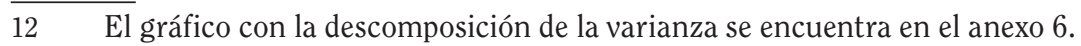




\section{CUADRO 7 \\ ESTIMACIONES: ENFOQUE ESTRUCTURAL}

\begin{tabular}{|c|c|c|c|c|c|c|}
\hline \multicolumn{7}{|c|}{ Coeficientes } \\
\hline Método & $\begin{array}{c}\operatorname{Inflacion}(\mathrm{t}-1) \\
\beta_{2}\end{array}$ & $\begin{array}{c}\text { Expectativas } \\
\beta_{3}\end{array}$ & $\begin{array}{c}\text { Brecha } \\
\beta_{4}\end{array}$ & $\begin{array}{c}\text { Constante } \\
\beta_{1}\end{array}$ & $\begin{array}{c}\text { Efecto } \\
\text { acumulado } \\
(\mathrm{pp})\end{array}$ & $\begin{array}{l}\text { Vida media } \\
\text { (meses) }\end{array}$ \\
\hline Efectos fijos & 0,8280 & 0,0040 & $-5,29 \mathrm{E}-14$ & $-0,0199$ & \multirow{2}{*}{5,8140} & \multirow{2}{*}{3,6725} \\
\hline (P-value) & $(0,0000)^{*}$ & $(0,0000)^{*}$ & $(0,2830)$ & $(0,0000)^{*}$ & & \\
\hline Efectos aleatorios & 0,8574 & 0,0035 & $-4,53 \mathrm{E}-14$ & $-0,1740$ & \multirow{2}{*}{7,0126} & \multirow{2}{*}{4,5053} \\
\hline (P-value) & $(0,0000)^{*}$ & $(0,0000)^{*}$ & $(0,3590)$ & $(0,0000)^{*}$ & & \\
\hline Arellano Bond & 0,8349 & 0,0050 & $-6,86 \mathrm{E}-14$ & $-0,0292$ & \multirow{2}{*}{6,0569} & \multirow{2}{*}{3,8414} \\
\hline (P-value) & $(0,0000)^{*}$ & $\left(0,0000^{*}\right)$ & $(0,0690)$ & $(0,0000)^{*}$ & & \\
\hline Arellano Bond (2steps) & 0,8350 & 0,0049 & $-6,86 \mathrm{E}-14$ & $-0,0290$ & \multirow{2}{*}{6,0606} & \multirow{2}{*}{3,8439} \\
\hline (P-value) & $(0,0000)^{*}$ & $(0,5800)$ & $(0,9090)$ & $(0,6550)^{*}$ & & \\
\hline $\begin{array}{l}\text { Regresiones } \\
\text { individuales** }\end{array}$ & 0,7595 & 0,0013 & $-6,21 \mathrm{E}-14$ & $-0,0227$ & \multirow[t]{2}{*}{4,1580} & \multirow[t]{2}{*}{2,5197} \\
\hline (P-value) & $(0,0000)^{*}$ & $(0,2003)$ & $(1,0000)$ & $(0,7405)^{*}$ & & \\
\hline
\end{tabular}

*Significativo al 1\%, **Resultado equiponderado.

Fuente: Elaboración propia con base en las estimaciones realizadas.

Los resultados de la estimación de los modelos de datos de panel muestran un alto grado de persistencia intrínseca $\left(\beta_{2}\right)$ contrastando con un bajo grado de persistencia extrínseca $\left(\beta_{4}\right)$ y una baja persistencia basada en expectativas $\left(\beta_{3}\right)$. Los coeficientes relacionados con la variable expectativa resultaron ser, a pesar de su magnitud, estadísticamente diferentes de cero. Los coeficientes asociados con la brecha del producto en ninguno de los métodos de estimación resultaron ser significativos.

CUADRO 8

ESTIMACIONES: ENFOQUE ESTRUCTURAL (CONTINUACIÓN)

\begin{tabular}{cccc} 
& \multicolumn{2}{c}{ Otras regresiones individuales } & \\
Grupos & Coeficiente** & Efecto acumulado (pp) & Vida media (Meses) \\
\hline General & 0,7127 & 3,4811 & 2,0468 \\
(P-value) & $(0,0000)^{*}$ & & 0,8054 \\
Sin servicios & 0,4229 & 1,7327 & 1,2120 \\
(P-value) & $(0,0000)^{*}$ & & 1,4989 \\
Sin regulados & 0,5644 & 2,2959 & \\
(P-value) & $(0,0000)^{*}$ & 2,7009 & \\
Sin servicios regulados & 0,6298 & & \\
(P-value) & $(0,0000)^{*}$ & & \\
\hline
\end{tabular}

*Significativo al 1\%, **Promedio calculado con los ponderadores del IPC Fuente: Elaboración propia con base en las estimaciones realizadas. 
Excluyendo los bienes más rígidos de la canasta del IPC, se evidencia que el nivel de persistencia inflacionaria disminuye. La reducción más grande de la persistencia ocurre cuando se excluyen los servicios del IPC. La reducción de la persistencia también sucede, pero en menor magnitud, cuando se excluyen los artículos regulados y los servicios regulados. La reducción de la persistencia al excluir estos artículos es congruente con la evidencia encontrada por Lunnenmann(2005) para la Unión Europea.

Los resultados de las estimaciones de la especificación propuesta por la ecuación 17, bajo el enfoque paramétrico de Marques (2004), se presentan en el cuadro 9. Los coeficientes estimados bajo los métodos de efectos fijos, efectos aleatorios, Arellano y Bond una etapa, y Arellano y Bond en 2 etapas, resultaron ser negativos. Chaverri y Torres (2010), y Marques(2004) mencionan que el valor debe ser positivo para que la persistencia sea un problema de interés, debido a que un signo negativo del parámetro indicaría que un choque tiene efectos contradictorios en la inflación ${ }^{13}$, por lo que un signo negativo de es interpretado como un grado de persistencia nulo.

El promedio ponderado de los coeficientes obtenidos mediante regresiones individuales resultó ser 0,1951, en el cálculo de este promedio se incluyeron coeficientes que resultaron ser negativos, así que si se toman los valores negativos como de persistencia 0 y se recalcula, se obtiene que el nivel de persistencia a nivel agregado que resulta ser 0,2605 un valor que continua siendo inferior al estimado bajo el esquema univariado.

CUADRO 9

ESTIMACIONES: ENFOQUE PARAMÉTRICO

\begin{tabular}{|c|c|c|c|}
\hline Método & Coeficiente * & Efecto acumulado & Vida media (Meses) \\
\hline Efectos fijos & $-0,1455$ & 0,87 & No aplica \\
\hline Efectos aleatorios & $-0,1354$ & 0,88 & No aplica \\
\hline Arellano Bond & $-0,0232$ & 0,98 & No aplica \\
\hline Arellano Bond (2steps) & $-0,0067$ & 0,99 & No aplica \\
\hline Regresiones individuales** & 0,1951 & 1,24 & 0,50 \\
\hline Regresiones individuales (Solo Positivos)** & 0,2605 & 1,35 & 0,50 \\
\hline
\end{tabular}

*Significativos al 1\%,**Promedio calculado con los ponderadores del IPC

Fuente: Elaboración propia con base en las estimaciones realizadas.

También bajo esta metodología la exclusión de artículos regulados, servicios y servicios regulados reduce el grado de persistencia inflacionaria.

\section{CONCLUSIONES}

En este documento se estimó el grado de persistencia inflacionaria, en esquemas tanto univariados como multivariados, $y$ se determinó el grado de rigidez de precio de los subíndices de precios regulados- no regulados y bienes- servicios.

$\overline{13}$ Si entonces las variaciones de los índices de precios convergen con oscilaciones a su valor de equilibrio. 
La evidencia apunta a que el comportamiento de los precios regulados y servicios tienen una mayor varianza que otros subíndices del IPC, además se encontró que estos artículos tienen un mayor grado de rigidez de precios.

También se encontró que dependiendo del tipo de ajuste del precio, es decir si es un aumento o una disminución, existen diferentes grados de rigidez. Se determinó que el 74\% de los artículos del IPC que tienen rigidez de precios presentan un mayor grado de rigidez a la disminución de sus precios.

Adicionalmente se detectó que, en promedio, las variaciones de precios de los bienes ocurren en una mayor magnitud en diciembre y los servicios en enero. Por otra parte las variaciones de los precios de los artículos regulados ocurren en una mayor magnitud en enero, abril y mayo.

De acuerdo con el enfoque paramétrico, se encontró que bajo un esquema univariado los grados de persistencia de las variaciones de los subíndices servicios y regulados son menores que el grado de persistencia estimado para las variaciones del IPC o los subíndices de bienes y no regulados. Esto sucede debido a que el ajuste de los precios de los servicios y regulados es escalonado.

Se encontró que bajo un esquema multivariado, dentro del enfoque CPNKH, que un choque en las variaciones los precios regulados se propaga a las variaciones de los precios no regulados y la brecha del producto. Además este choque genera efectos significativos durante 4 meses sobre las variaciones del IPC con un efecto máximo de 0,61pp en el segundo mes después del choque inicial, $y$ a la vez generando una respuesta más persistente que un choque en las variaciones de los precios no regulado. Las estimaciones de la descomposición de la varianza muestran que las variaciones de los precios regulados aportan un porcentaje considerable de variabilidad a las variables expectativas de inflación, brecha del producto y a las variaciones de los precios no regulados.

De acuerdo con el enfoque CPNKH, utilizando datos desagregados, se identificó que la persistencia intrínseca es la que se presenta en mayor magnitud. Por otra parte, bajo el enfoque paramétrico las estimaciones del esquema univariado propuesto por Robalo Marques muestran un grado menor de persistencia, con datos agregados se estima un grado de persistencia de 0,2939 y con datos desagregados se estima en 0,2605.

Por último se encontró que al excluir los artículos con mayores grados de rigidez, es decir los artículos con precios regulados y servicios, el grado de persistencia tiende a reducirse.

Entre ambos enfoques subyacen diferencias en los resultados de persistencia. Bajo el enfoque paramétrico, univariado, los grados de persistencia son menores con respecto a el enfoque CPNKH; esto debido a que el enfoque paramétrico no considera la propagación, retroalimentación y dinámica de los componentes del IPC ante choques. Ante esta situación cobra relevancia el estudio de la persistencia de las respuestas de los componentes del IPC ante perturbaciones, y no así la persistencia de estas perturbaciones por si mismas, como lo indican los resultados obtenidos mediante la estimación del Vector Auto Regresivo.

Los ajustes de los precios regulados representan un riesgo para el cumplimiento del objetivo inflacionario, esto debido que un choque de las variaciones de los precios regulados puede fácilmente sacar a la inflación de su rango meta, y ya que propaga a las expectativas de inflación podría provocar efectos de segunda ronda. Por lo que, como recomendación, se debe revisar la metodología de fijación tarifaria aplicada por la Autoridad Reguladora de los Servicios Públicos ${ }^{14}$, esto para indexar los ajustes de los precios regulados a la inflación, y evitar divergencias entre los subíndices de precios regulados y no regulados.

En otras consideraciones de política monetaria, la existencia de rigidices de precios y persistencia inflacionaria indican que el Banco Central deberá responder ajustando su instrumento en una mayor magnitud ante perturbaciones sobre la inflación, para lograr su objetivo, Esto implica un mayor costo en términos de la brecha del producto al reducir la inflación de la economía. 


\section{REFERENCIAS}

Altissimo, F., Erhmann, M., \& Smets, F. (2006). Inflation persistence and price-setting behavior in the euro area (Working paper No.46). European Central Bank.

Babecký, J., Coricelli , F., \& Horváth , R. (2008). Assessing inflation persistence: Micro evidence on an inflation target economy. Prague: CERGE-IE.

Baltagi , B. H. (2005). Econometric analysis of panel data. Great Britain: Wiley.

Banco Central de Costa Rica. (Marzo-2013). Informe mensual de coyuntura económica. División económica.

Bilke, L. (2005). Break in the mean an persistence of inflation: A sectoral analisis of French CPI (Working paper No.463). European Central Bank.

Bilke, L. (2005). Break in the mean an persistence of inflation: A sectorial analisis oh french CPI (Working paper No.463). European Central Bank.

Bils, M., \& Klenow, P. (2002). Some evidence on importance of sticky prices (Working Paper No.9069). NBER .

Blinder , A. (1991). Why are prices sticky? (Working Paper No 3646). NBER.

Calvo , G. (1983). Staggered prices in a utility-maximizing frameword. Journal of Monetary Economics, 383-398.

Chaverri, C., \& Torres, C. (2010). Dinámica inflacionaria y persistencia en Costa Rica: 1953-2009. DEC-DIE-025-2010, Banco Central de Costa Rica.

De Gregorio, J. (2007). Macroeconomía: Teoría y políticas. México: Pearson Education.

Estrella, A., \& Fuhrer, J. (1998). Dynamic inconsistencies: Counterfactual implications of a class of rational expectations models. Working Paper 98-5, Federal Reserve Bank of Boston.

Gagnon , E. (2009). Price setting during low and high inflation: Evidence for Mexico. The Quarterly Journal of Economics, 1221-1263.

Galí, J., Angeloni , I., Ehrman , M., Aucremanne, L., Levin, A., \& Smets, F. (2006). New evidence on inflation persistence and price stickiness in the euro area: Implications for macro modeling. Journal of European Economic Association , 562-574.

Ghysels, E. (1990). Unit-Root Tests and the Statistical Pitfalls of Seasonal Adjustment: The Case of U.S. PostwarReal Gross National Product. Journal of Business \& Economic Statistics 8(2), 145-152.

Ghysels, E., \& Perron , P. (1993). The Effect of Seasonal Adjustment Filters on Tests for a Unit Root. Journal of Econometrics 55, 57-98.

Hansen, L. P., \& Sargent, T. J. (1993). Seasonal and aproximation errors in rational expectation models. Journal of Econometrics, 21-55.

Kasuya, M. (1999). Downward price rigidity of the Japanese CPI: Analysis by probability density function and spatial density functions (Working Paper 99-3). Research and statistics, Bank of Japan.

Le Bihan , H., \& Matheron, J. (2011). Price stickiness and sectoral inflation: Additional evidence (Working Paper No.353). Banque de France.

Le Bihan, H., \& Matheron , J. (2011). Price stickiness and sectorial inflation: Additional evidence (Working Paper No.353). Banque de France .

Lee, Y.-J. (2006). Diagnostic testing for dynamic panel data models. Department of Economics, Indiana University.

León , J. (2012). A disagregate model and second round effects for the CPI in Costa Rica. DEC-DIEDI-10-2012, Banco Central de Costa Rica.

López, E. (2008). Algunos hechos estilizados sobre el comportamiento de los precios regulados en Colombia (Documento de Trabajo No.527). Banco de la República de Colombia . 
Lunnemann , P., \& Mathä, T. (2005). Regulated and services prices and inflation persistence (Working Paper No.466). European Central Bank .

Lunnemann, P., \& Mathä, T. (2004). How persistent is disaggregate inflation?: An analysis across EU15 countries an d HICP sub-indices (Working Paper No.415). European Central Bank.

Mac Donald, R., \& Wójcik, C. (2003). Catching up: The role of demand, supply and regulated prices effects on the real exchange rate of four accession countries (Working Paper No.899). CESinfo.

Marques, C. R. (2004). Inflation persistence: Facts or artefacts? (Working Paper No.371). European Central bank .

Menz, J. O., \& L. V. (2009). A detailed derivation of the sticky price and sticky information New Keynesian DSGE model. DEP discussion paper, University of Hamburg.

Nakamura, E., \& Steinsson, J. (2013). Price rigidity: Microeconomic evidence and macroeconomic implications. Columbia University .

Raciborski , R. (2008). Searching for additional sources of inflation persistence: The micro price panel data approach (Working Paper No.132). National Bank of Belgium.

Rodríguez, A. (2012). Propagación de choques inflacionarios en Costa Rica. Documento de investigación No.09-2012, Banco Central de Costa Rica.

Segura Rodríguez, C., \& Vásquez Carvajal , J. (2011). Estimación del parámetro de suavizamiento del filtro de Hodrick y Prescott para Costa Rica. DEC-DIE-DT 006-2011, Banco Central de Costa Rica.

Torres, C. (2012). Costa Rica: Determinación de Cambios estructurales en el nivel de la tasa de inflación: Periodo 1997-2011. Documento de Trabajo 02-2012, Banco Central de Costa Rica. 


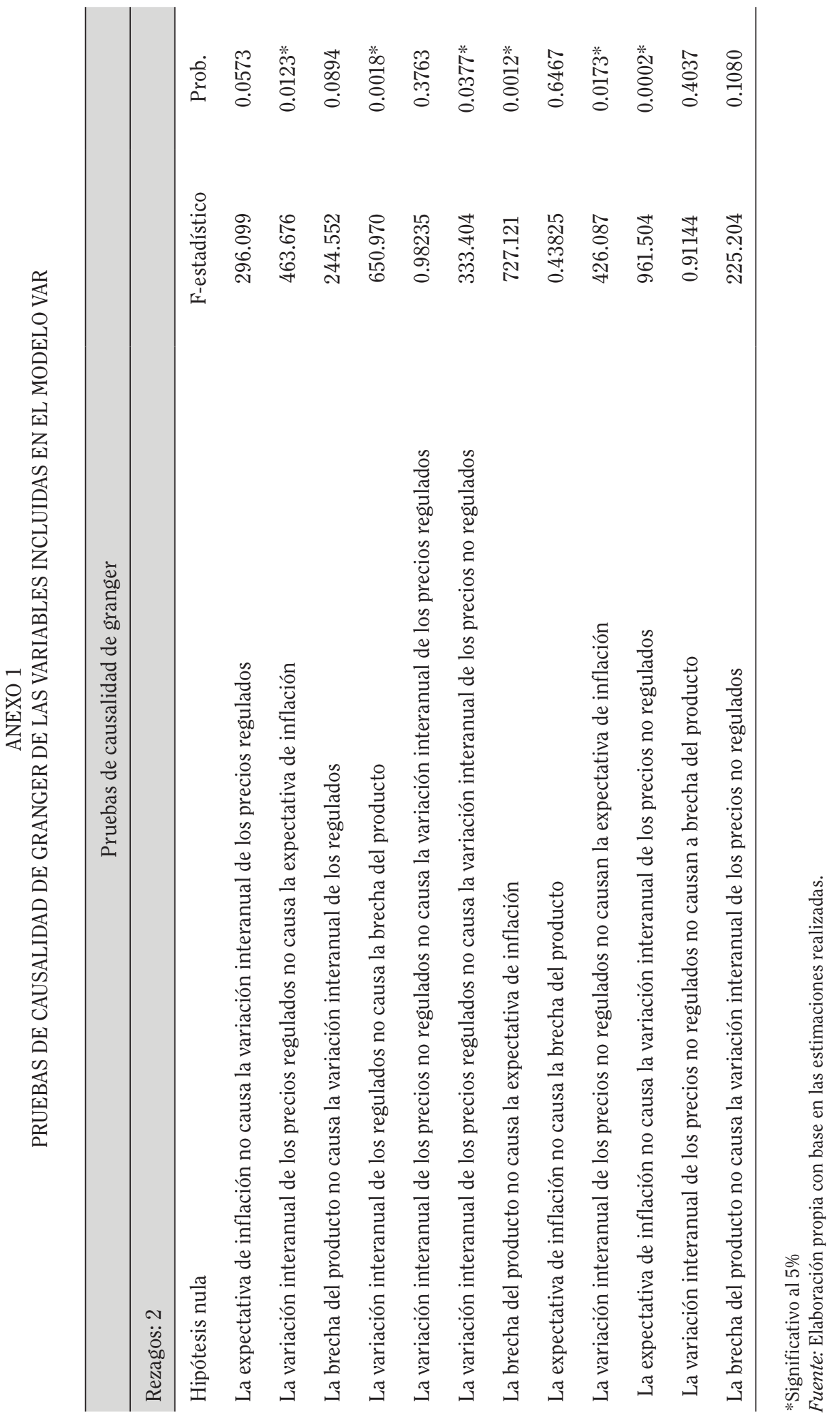




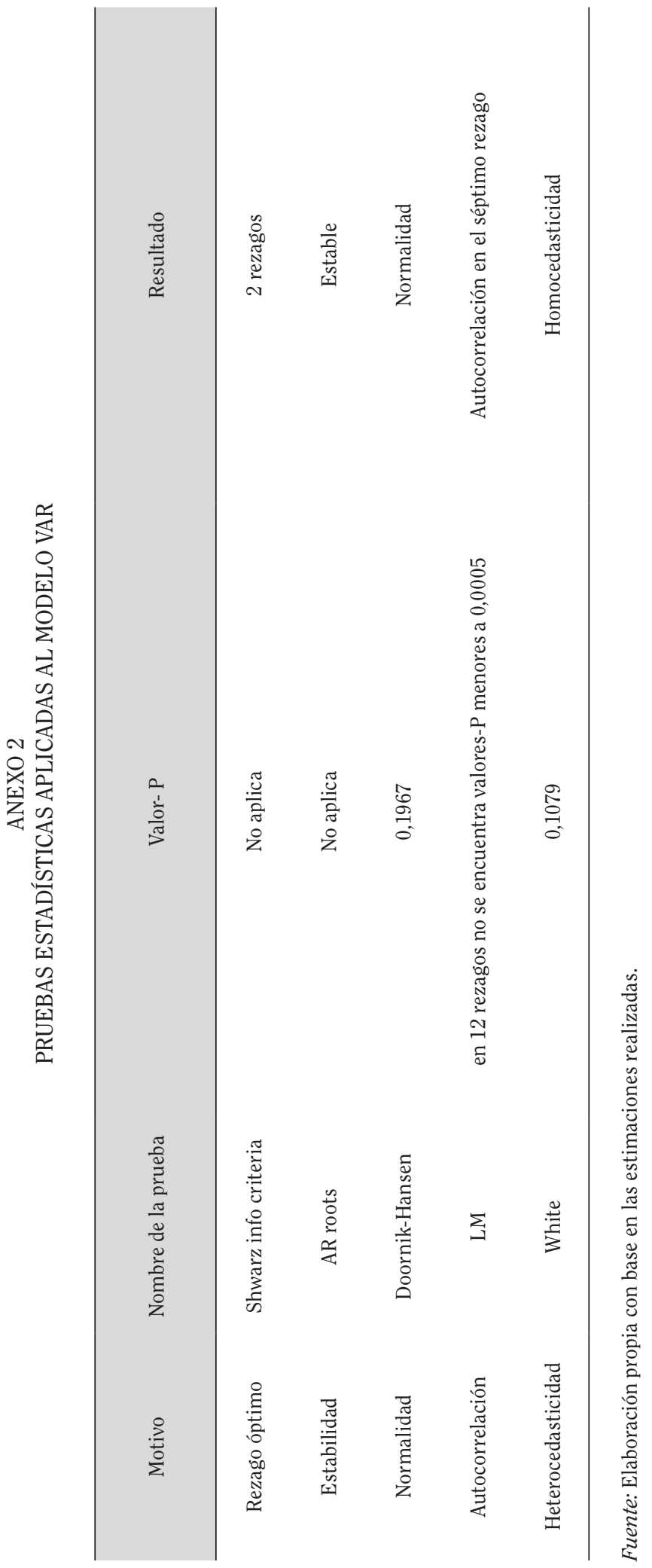


ANEXO 3

FUNCIONES IMPULSO RESPUESTA (GRÁFICOS COMBINADOS)
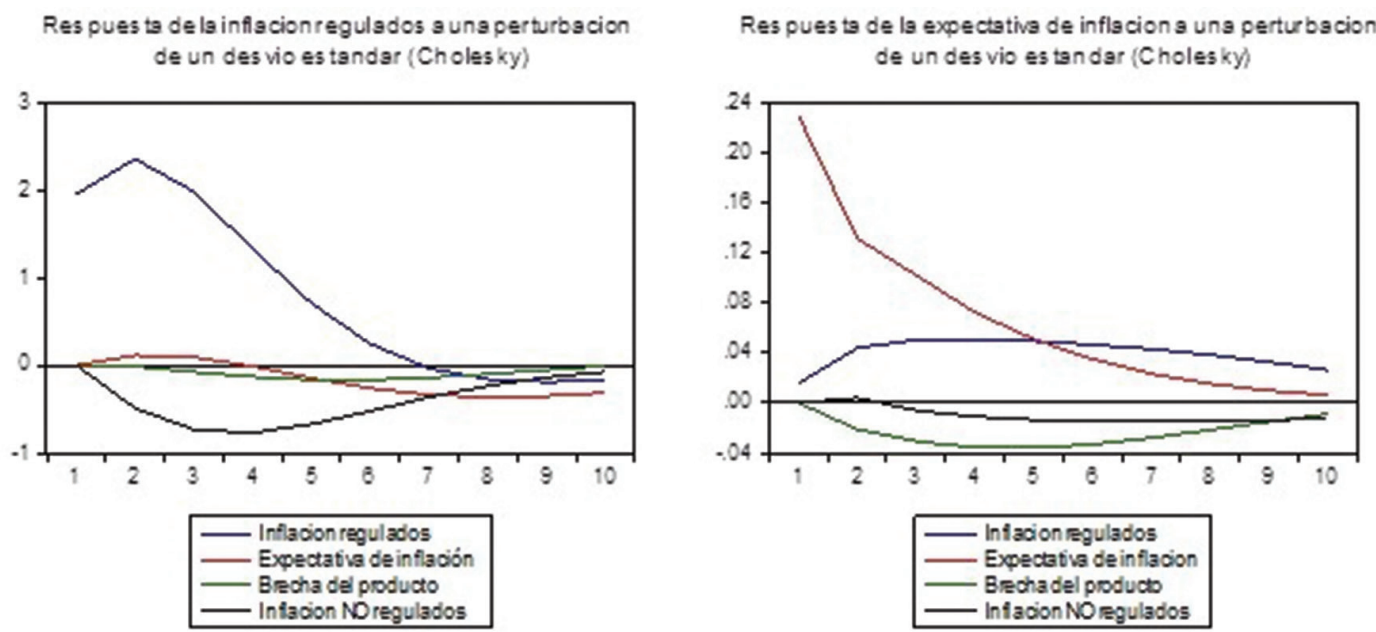
de un des vio estandar (Choles $k y$ )

Res pues ta de la brechs del producto s ung perturbacion de un des vio es tender (Cholesky)
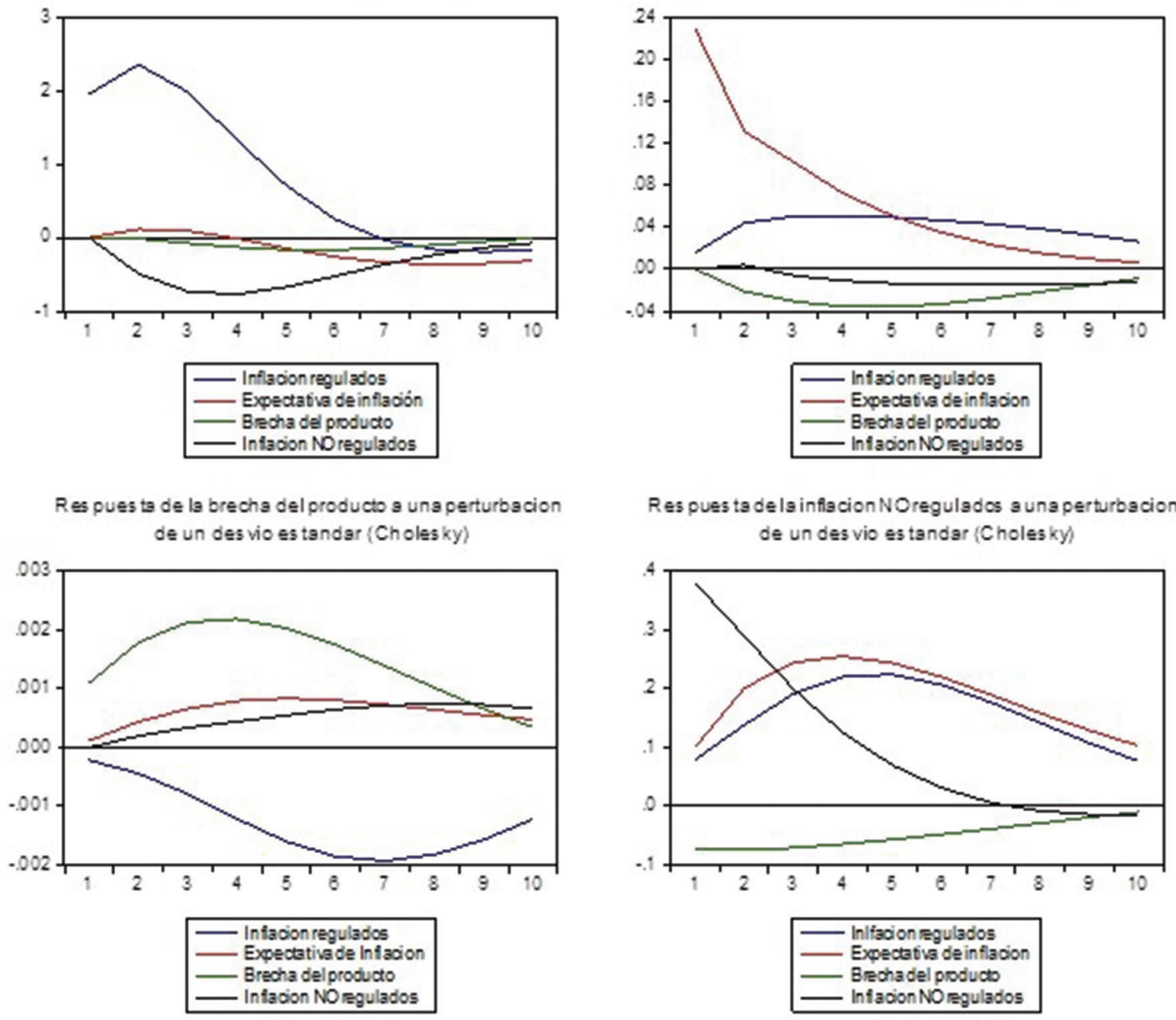

Res pues ta dels inflecion NOregulados sung perturbacion de un des vio es tander (Choles $k y$ )

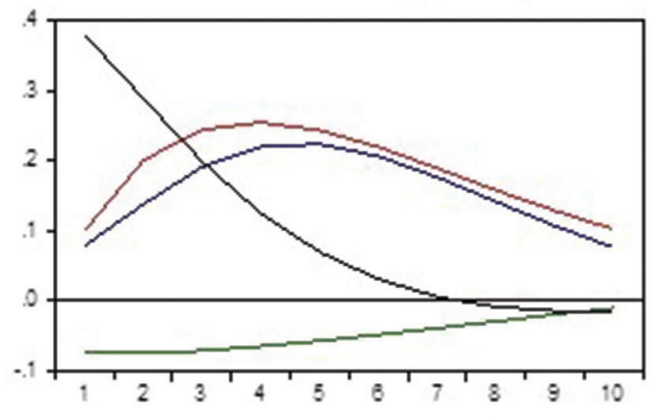

Inlfacion regulados Expectativa de inflacion Brechadel products Infacion NO regulados

Fuente: Elaboración propia con base en las estimaciones realizadas. 


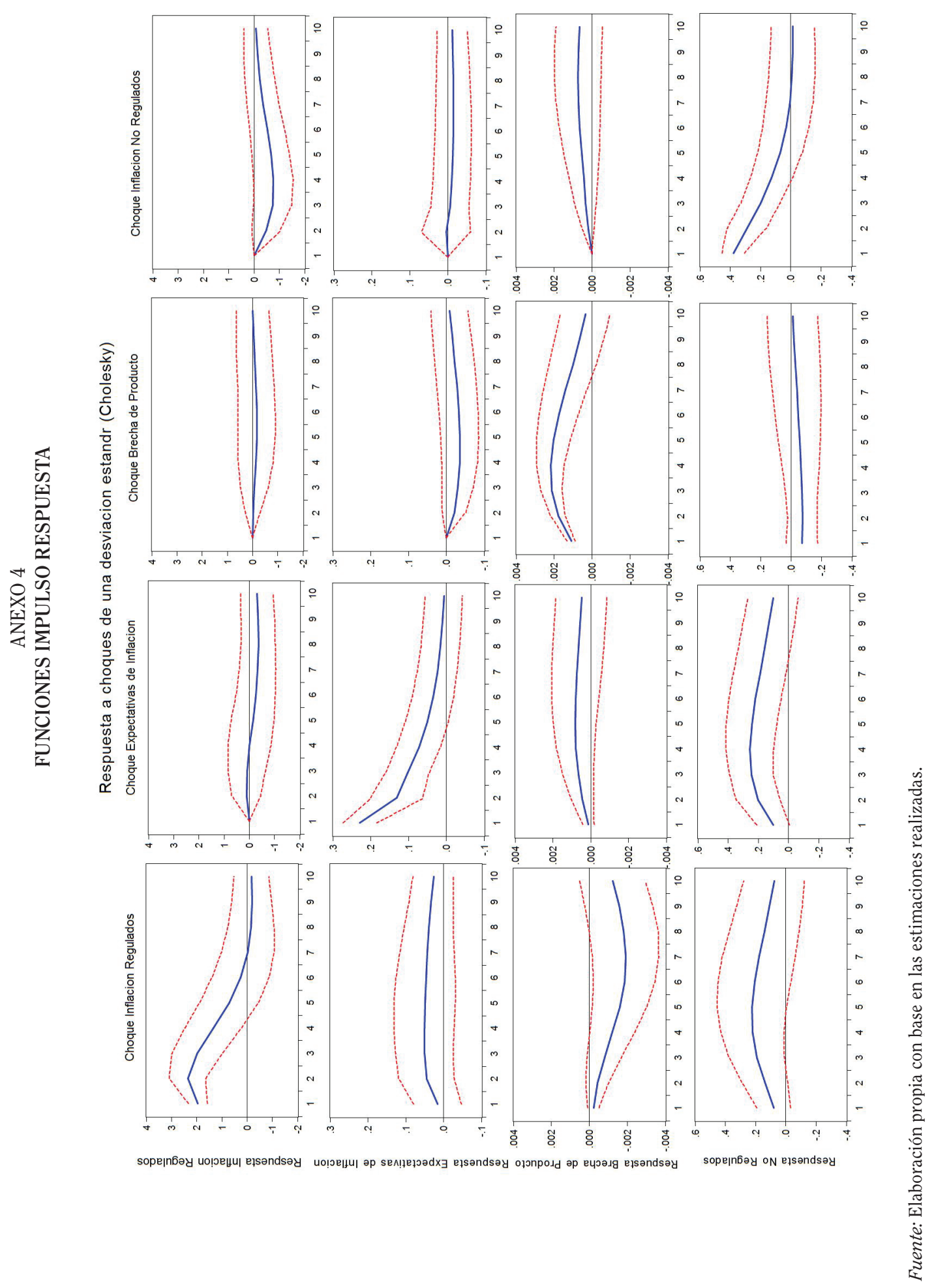



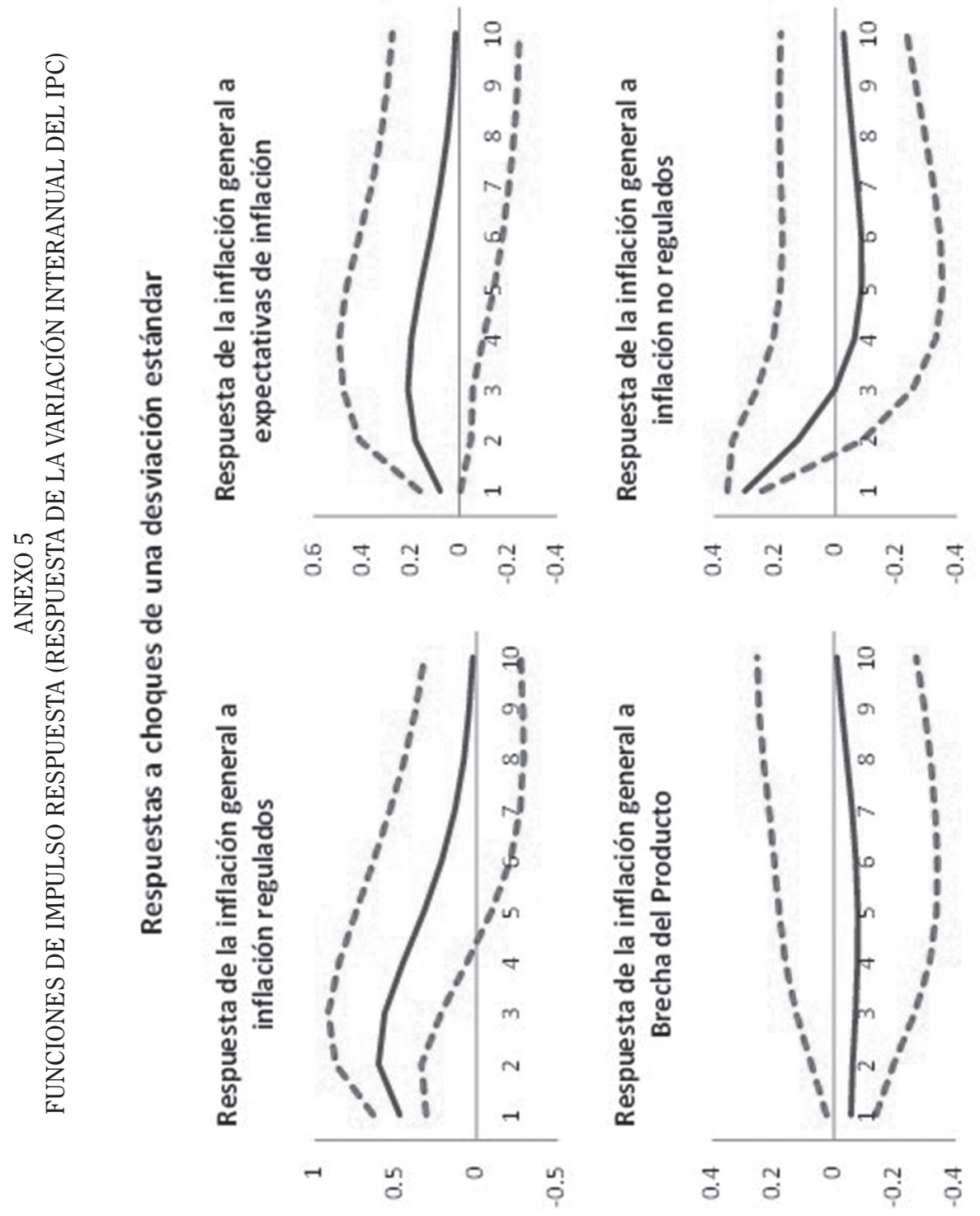

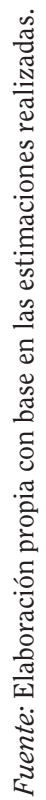



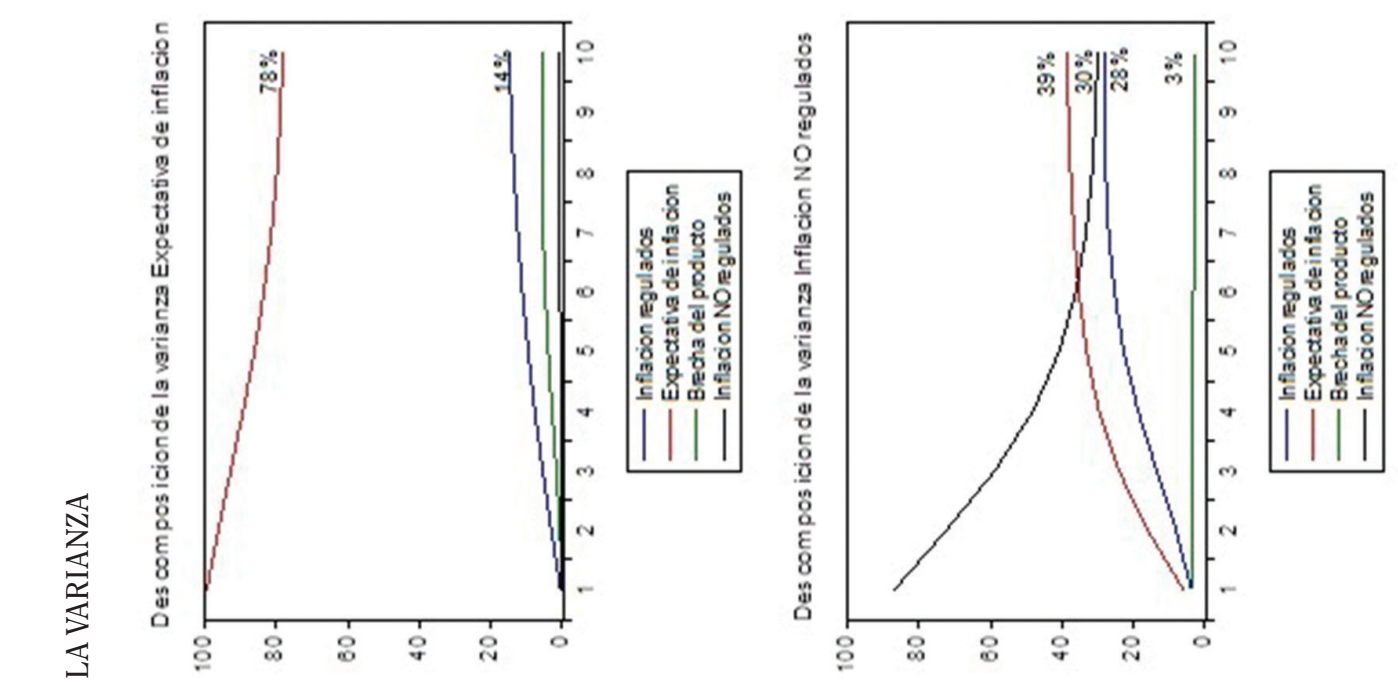

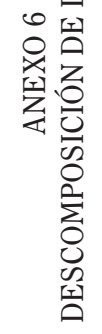
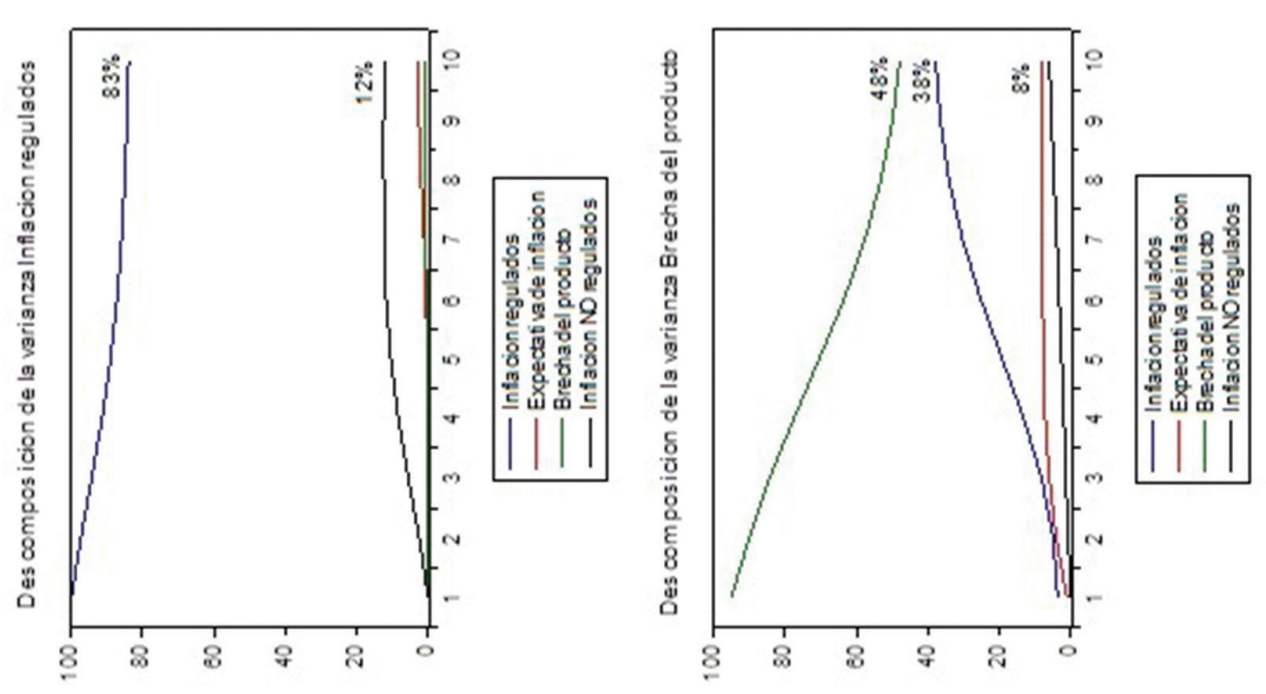

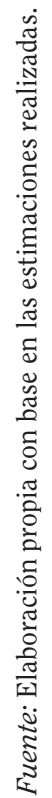




\section{ANEXO 7 \\ MECANISMOS DE FIJACIÓN DE PRECIOS EN COSTA RICA}

Las metodologías de fijación tarifaria de la Autoridad Reguladora de los Servicios Públicos (ARESEP), en su mayoría responden a modelos contables basados en una regulación de tasa de retorno, como muestra el cuadro 8. Esto es congruente al principio de servicio al costo (Ley \# 7593), el cual plantea que la ARESEP debe reconocer los costos asociados con la prestación del servicio y además permitir una retribución adecuada y competitiva a los prestadores del servicio.

CUADRO 8

TIPOS DE REGULACIÓN POR ARTÍCULO REGULADO

\begin{tabular}{rlll}
\hline & Artículo & Método de regulación & Regulador \\
\hline 1 & Arroz & Estructura de costos & MEIC \\
2 & Servicio de agua & Estructura de costos & ARESEP \\
3 & Limpieza de vías & - & - \\
4 & Recolección de basura & - & - \\
5 & Servicio de electricidad & Tasa de retorno & ARESEP \\
6 & Gas licuado & Ajuste indexado/ Tasa de retorno & ARESEP \\
7 & Gasolina & Ajuste indexado/ Tasa de retorno & ARESEP \\
8 & Diésel & Ajuste indexado/ Tasa de retorno & ARESEP \\
9 & Revisión técnica de vehículo & - & ARESEP \\
10 & Licencia de conducir & - & - \\
11 & Pasaje en autobús urbano & Tasa de retorno & ARESEP \\
12 & Pasaje en autobús interurbano & Tasa de retorno & ARESEP \\
13 & Transporte en taxi & Tasa de retorno & ARESEP \\
14 & Servicio de teléfono celular & Precio máximo & SUTEL \\
15 & Servicio de teléfono fijo & Precio máximo & SUTEL \\
\hline
\end{tabular}

Fuente: Elaboración propia con base en las estimaciones realizadas.

La principal desventaja que reconoce la literatura sobre la metodología de tasa de retorno es que no incentiva la eficiencia en las empresas reguladas, esto debido a que la fijación tarifaria reconoce sus costos sin analizar su eficiencia, por lo que los incrementos de los costos se traducen en un incremento de los precios. Además como desventaja adicional la literatura reconoce que la aplicación de esta metodología requiere de volúmenes de información que por naturaleza están en poder del regulado. Lo que genera un problema de información asimétrica que pone en ventaja al regulado. 
ANEXO 8

ANÁLISIS DE COINTEGRACIÓN DE LOS ÍNDICES DE PRECIOS REGULADOS Y NO REGULADOS

Siguiendo la teoría de Calvo (1983) los ajustes de los precios rígidos, en este caso los regulados, son escalonados y se ajustan alrededor de un índice general de precios, de manera que cuando el agente fijador de precios recibe la señal de reoptimizar sus precios toma en cuenta sus costos como la inflación observada y esperada. Si este tipo de ajuste ocurre las series de precios regulados y precios no regulados deben cointegrar.

En este apartado se prueba la hipótesis de cointegración entre los índices de precios regulados $y$ no regulados esto con el fin de identificar un patrón de convergencia o divergencia entre los dos índices, la figura 1 representa en el panel (a) el ajuste de precios escalonado cointegrante bajo la hipótesis de un ajuste "a la Calvo", el panel (b) muestra un ajuste de precios escalonado pero divergente, es decir que no toma en cuenta la inflación observada y esperada.

FIGURA 1

TRAYECTORIAS HIPOTÉTICAS DE LOS ÍNDICES DE PRECIOS REGULADOS Y NO REGULADOS
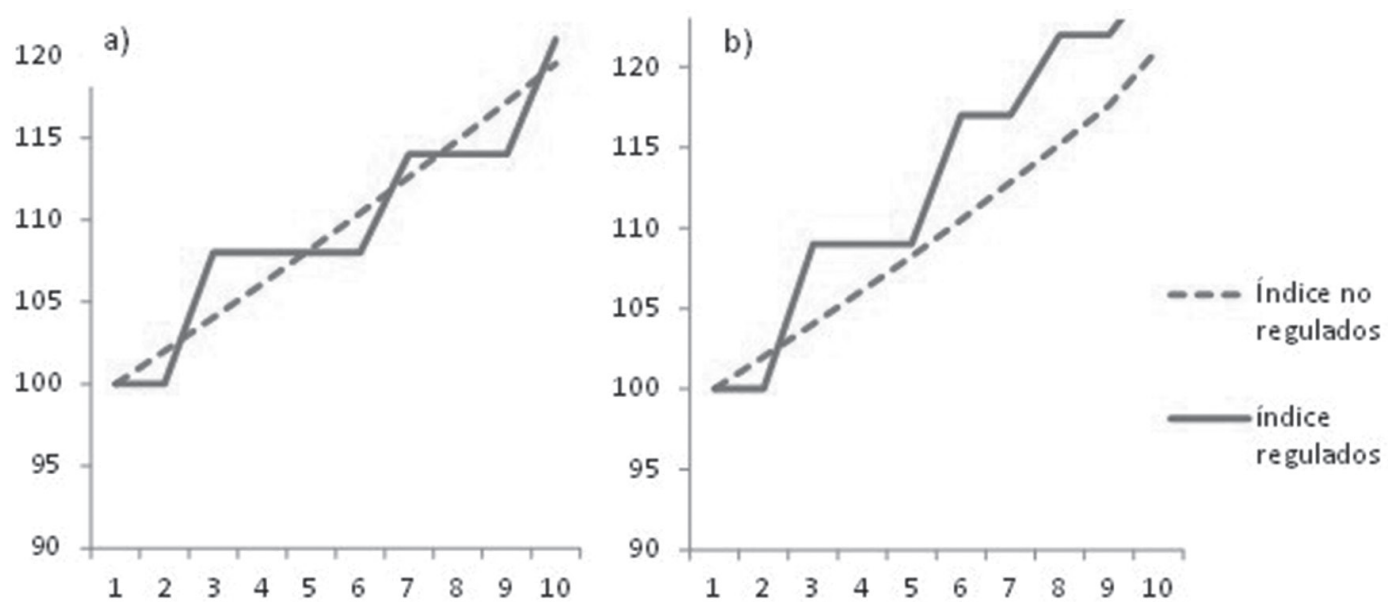

Fuente: Elaboración propia.

El ajuste representado por el panel (a) de la figura 1 es el caso de la regulación idónea, porque los precios de los artículos regulados se ajustan a los de la economía y no viceversa.

El ajuste de los precios regulados representado por el panel (b) es el caso donde el fijador de precios sobre reacciona ante las variaciones de los precios no regulados y provoca "Círculos inflacionarios", esto se hace referencia a una situación donde los incrementos de los precios de los artículos regulados incrementan los costos de producción de artículos cuyos precios no están regulados. Al incrementar los costos de producción las empresas trasladarán una parte de este incremento a sus precios y otra a mejoras en su productividad, luego el incremento de los precios de los artículos no regulados generan un incremento los costos de artículos regulados, pero si su regulación es de tasa de retorno las empresas trasladaran este incremento de costos a un nuevo ajuste tarifario repitiendo el ciclo.

Un análisis del orden de integración de los índices de precios regulados y no regulados (Cuadro 9) revela que estos índices son integrados de orden 1. 
Siguiendo la metodología de Granger se estimó una combinación lineal de los índices, por lo que las variables fueron ordenadas por su grado de exogeneidad.

$$
\text { 1. } I_{t}^{\text {No regulados }}=\beta_{1}+\beta_{2} I_{t}^{\text {Regulados }}+\varepsilon_{t}
$$

La relación entre ambos índices es cointegrante si y solo si la variable es estacionaria en media, varianza y covarianza. Los resultados obtenidos de las pruebas de integración de los residuos reflejan que son no estacionarios por lo que no existe una relación de equilibrio de largo plazo en los índices.

CUADRO 9

PRUEBAS DE RAÍZ UNITARIA: AUGMENTED DICKEY-FULLER

\begin{tabular}{cccc}
\hline & & Valor-P & \\
\hline & tendencia e intercepto & intercepto & ninguno \\
\hline Índice regulados: & & & \\
Nivel & 0,5827 & 1 & 1 \\
Primer diferencia & 0 & 0 & 0 \\
Índice no regulados. & 0,6106 & 0,9985 & 1 \\
Nivel & 0 & 0 & 0,3871 \\
Primer diferencia & & & \\
Residuos & 0,0206 & 0,0717 & 0,0013 \\
Nivel & 0 & 0 & 0 \\
Primer diferencia & &
\end{tabular}

Fuente: Elaboración propia con base en las estimaciones realizadas.

Como un segundo criterio se aplicó la prueba de Johansen (Cuadro 10), la que evidenció que no hay suficiente evidencia estadística para rechazar la hipótesis nula de que al menos existe un vector de cointegración.

CUADRO 10

PRUEBA DE COINTEGRACION DE JOHANSEN, ÍNDICES REGULADOS Y NO REGULADOS

\begin{tabular}{ccccc}
\hline & \multicolumn{2}{c}{ Test de co-integración (Traza) } & \\
\hline Hipótesis & & Trace & 0.05 \\
Número de vectores co-integrantes & Valor característico & Estadístico & Valor critico & Prob. \\
Ninguno & 0.055891 & 1.836 .320 & 2.587 .211 & 0.3200 \\
Al menos 1 & 0.030715 & 6.457 .762 & 1.251 .798 & 0.4045 \\
\hline Hipótesis & Test de co-integración (Valor propio máximo) & & Prob. \\
Número de vectores co-integrantes & Valor característico & Valor propio máximo & 0.05 & 0.4240 \\
Ninguno & 0.055891 & estadístico & Valor Critico & 0.4045 \\
Al menos 1 & 0.030715 & 6.457 .762 & 1.938 .704 & 1.251 .798 \\
\hline
\end{tabular}

Fuente: Elaboración propia con base en las estimaciones realizadas. 
En el caso de Costa Rica la no cointegración entre los dos índices es evidencia que apunta a que los precios regulados no se ajustan a la inflación, sino que generan círculos inflacionarios, esto porque la divergencia entre los dos índices ocurre debido a que los ajustes de precios de los artículos regulados se dan mediante tasas de crecimiento que son mayores que las tasas de crecimiento de los precios del resto de artículos. 
